\title{
Detecting Arbitrary Stable Properties Using Efficient Snapshots
}

\author{
Ajay Kshemkalyani, Senior Member, IEEE, and Bin Wu
}

\begin{abstract}
A stable property continues to hold in an execution once it becomes true. Detecting arbitrary stable properties efficiently in distributed executions is still an open problem. The known algorithms for detecting arbitrary stable properties and snapshot algorithms used to detect such stable properties suffer from drawbacks such as the following: They incur the overhead of a large number of messages per global snapshot, or alter application message headers, or use inhibition, or use the execution history, or assume a strong property such as causal delivery of messages in the system. We solve the problem of detecting an arbitrary stable property efficiently under the following assumptions: P1) The application messages should not be modified, not even by timestamps or message coloring. P2) No inhibition is allowed. P3) The algorithm should not use the message history. P4) Any process can initiate the algorithm. This paper proposes a family of nonintrusive algorithms requiring $6(n-1)$ control messages, where $n$ is the number of processes. A three-phase strategy of uncoordinated observation of local states is used to give a consistent snapshot from which any stable property can be detected. A key feature of our algorithms is that they do not rely on the processes continually and pessimistically reporting their activity. Only the relevant activity that occurs in the thin slice during the algorithm execution needs to be examined.
\end{abstract}

Index Terms-Distributed system, global state, stable predicate, stable property, distributed snapshot.

\section{Introduction and Problem Definition}

\subsection{Problem Formulation}

A snapshot of a distributed system represents a consistent global state of the system [4]. Recording distributed snapshots of an execution is a fundamental problem in asynchronous distributed systems and is used for observing various properties of interest [1], [2], [4], [7], [11], [14], [16], [19], [22], [23], [25].

A restricted class of properties, termed stable properties, are monotonic in the following sense: A stable property continues to hold in an execution once it becomes true [4]. Commonly occurring examples of stable properties are deadlock, termination, and system garbage. Many specialized algorithms are tailored to specific stable properties, such as to detect deadlock or termination or to perform garbage collection; see [17], [21] for a survey of such algorithms. However, our objective is to devise an efficient algorithm to detect an arbitrary stable property. While global snapshot algorithms can always be used to detect stable properties, they suffer from some or all of the following drawbacks: They are expensive and generally require a large number of messages per snapshot [4], [23], [22], [25], [7], [14], [16], or they use freezing of the application execution [7], or they alter application message headers [14], [16], [19], or they assume some strong properties such as causal message delivery in the system [1], [2].

- The authors are with the Department of Computer Science, University of Illinois at Chicago, 851 South Morgan Street, Chicago, IL 60607.

E-mail: ajayk@cs.uic.edu,bwu3@uic.edu.

Manuscript received 30 Apr. 2005; revised 10 Feb. 2006; accepted 22 Jan. 2007; published online 13 Feb. 2007.

Recommended for acceptance by R. Schlichting.

For information on obtaining reprints of this article, please send e-mail to: tse@computer.org, and reference IEEECS Log Number TSE-0116-0405.

Digital Object Identifier no. 10.1109/TSE.2007.1000.
Consider a distributed system that is modeled as a directed graph $(N, L)$, where $N$ is the set of processes and $L$ is the set of links connecting the processes. Let $n=|N|$ and $l=|L|$. We solve the open problem of efficiently detecting an arbitrary stable property under the following assumptions (P1-P4):

P1. The application messages should not be modified, not even by timestamps [6], [18] or message coloring.

P2. No freezing or inhibition [5] in the application execution is allowed. This rules out all three forms of inhibition: inhibition of send events, inhibition of receive events, and inhibition of local noncommunication events, i.e., internal events.

P3. The algorithm should not use the log of the message history.

P4. Any process can initiate the algorithm.

The known algorithms for detecting stable properties can detect only a subclass of stable properties-such as locally stable properties [17], strongly stable properties [14], and strong stable properties [21]—and may not satisfy all of (P1-P4).

\subsection{Prior Work}

A property is an abstract characteristic of an execution. An explicit formulation of a property in terms of process states and variables is a predicate. For example, deadlock is a property. A deadlock predicate is its explicit formulation, in terms of the blocked status variable, outgoing wait-for edge variables, and incoming wait-for edge variables at each process. Keeping this association in mind, we will henceforth use the term "predicates" rather than "properties." There are only the following algorithms that try to detect general stable predicates that are not tailored for a specific predicate:

- The algorithm by Marzullo and Sabel [17] can detect only locally stable predicates. A locally stable predicate 
is a stable predicate such that, once it becomes true, none of the variables used in the predicate formulation changes in value. Stated differently, the predicate can be determined only from the states of processes and no process involved in the predicate can change its state once the predicate holds.

The algorithm by Marzullo and Sabel requires the use of a (modified) vector clock [6], [18] that modifies application messages. The entire log of the execution history (of events that can affect the predicate) is implicitly used by a pessimistic sending condition followed by the processes. Specifically, the predicate detection algorithm receives the local histories from each process since the last time they were reported. It then computes the latest consistent subcut, which is defined as the collection of the latest reported states of processes that are consistent with each other. The predicate is evaluated for this latest consistent subcut. The protocol is sound-if $\phi$ holds for the latest consistent subcut, $\phi$ holds thenceforth. To show completeness, let $G S_{\phi}^{f}$ be the first global state in which $\phi$ holds, and let the local state at $P_{i}$ in this global state be $G S_{\phi}^{f}[i]$. As $\phi$ is locally stable, all events that happen after $G S_{\phi}^{f}[i]$ are not relevantthis is the key here-and $P_{i}^{\prime}$ 's state will not change further. As a result, no message sent after $G S_{\phi}^{f}[i]$ can cause inconsistency. Once $G S_{\phi}^{f}[i]$ is collected (for each $P_{i}$ ), the $G S_{\phi}^{f}[i]$ will appear on a latest consistent subcut and the locally stable predicate will be detected. The protocol implicitly assumes that, once the predicate $\phi$ becomes true, the involved processes will stop sending messages to report local activities, without actually knowing that $\phi$ has become true.

Termination, deadlock, and global virtual time can be formulated as locally stable predicates. The predicate "there are at most $k$ tokens" is not locally stable, as shown in [17]. In an execution where variables $x_{1}, \ldots x_{k}$ at processes $P_{1}, \ldots P_{k}$, respectively, take nondecreasing positive values, the predicate $x_{1} \times \ldots \times x_{k}>c$ can be seen to be not locally stable.

The protocol [17] is then used to derive even simpler protocols for deadlock and other locally stable properties, which are instantiations of the general protocol.

- The algorithm by Schiper and Sandoz [21] can detect only strong stable predicates. They define a consistent projection of a cut as a subcut containing those local process states that are mutually consistent with each other. A strong stable predicate is a stable predicate that can always be evaluated on the consistent projection of any global state, consistent or not. It is sufficient to evaluate the predicate on a consistent projection of any global state to determine if the predicate is true for the execution [21]. The algorithm implicitly assumes that, once the predicate becomes true (for a consistent projection of a cut over the "minimal" number of processes necessary), the involved processes will stop sending messages to report local activities without actually knowing that the predicate has become true.

In the basic version of the Schiper-Sandoz algorithm, processes continually report their new states to a central process that keeps evaluating consistent projections of the latest constructed global state until the evaluation leads to true. Thus, all processes follow a pessimistic sending rule as in the MarzulloSabel algorithm. The algorithm requires the use of a (modified) vector clock that modifies application messages to construct the consistent projections. The entire $\log$ of the execution history (of events that can affect the value of the predicate) is also implicitly used to construct the consistent projections for all global states, in order to detect this class of stable predicates. Specifically, "each process furnishes an unbounded sequence of local contributions ... (until termination or predicate detection)" [21].

The protocol is sound-if $\phi$ holds for the latest consistent projection, $\phi$ holds thenceforth. To show completeness, observe that once $\phi$ holds, by definition, the predicate will evaluate to true on the consistent projection of the first global state when $\phi$ holds.

Termination and deadlock can be formulated as strong stable predicates. Distributed garbage is not strong stable, as shown in [21].

- Lai and Yang [14] defined the class of strongly stable predicates. A strongly stable predicate is a stable predicate such that, if the predicate is true on some cut, consistent or not, the predicate must remain true for all subsequent cuts.

- $\quad$ Based on the Ho-Ramamoorthy two-phase deadlock detection algorithm [10], Kshemkalyani-Singhal gave a two-phase technique [12] that showed how to correctly detect deadlocks and how to detect stable properties. A possibly inconsistent snapshot of the execution is taken; if the local predicates indicate a deadlock, a second possibly inconsistent snapshot is initiated. If the same deadlock also holds in this second snapshot, it is concluded that deadlock indeed exists because there is an instant of time between the two snapshots when the deadlock also held. A generalized method to detect stable properties was then outlined [12]. While all locally stable predicates can be detected satisfying our assumptions (P1-P4) and without assuming FIFO channels, it is not clear how arbitrary local predicates would be detected.

- Recently, Atreya et al. [3] proposed an algorithm to detect a locally stable predicate by repeatedly taking possibly inconsistent snapshots as long as 1) there is one consistent state between the two possibly inconsistent snapshots, and 2) the values of the variables do not change in the two snapshots. This is, in essence, the Ho-Ramamoorthy algorithm [10] as modified and corrected in [12]. The generalization to detect locally stable properties other than deadlock was more formally shown than in [12]. Only locally stable predicates can be detected. 
TABLE 1

Comparing Algorithms to Detect Stable Predicates

\begin{tabular}{|c|c|c|c|c|}
\hline & Marzullo \& Sabel [17] & Schiper \& Sandoz [21] & Proposed v.1 & Proposed v.2 \\
\hline $\begin{array}{l}\text { Detectable } \\
\text { predicates }\end{array}$ & locally stable & strong stable & locally stable & all stable \\
\hline $\begin{array}{l}\text { Overhead at } \\
\text { nodes } \\
(=\text { control } \\
\text { msg. overhead) }\end{array}$ & $\begin{array}{l}\text { vector clock, } O(n)+ \\
\text { log of msgs \& events } \\
\text { w/timestamps }\end{array}$ & $\begin{array}{c}\text { vector clock, } O(n)+ \\
\text { log of msgs \& events } \\
\text { w/timestamps }\end{array}$ & $\begin{array}{c}- \\
\text { events log in slices } \\
\text { only during 3-phase }\end{array}$ & $\begin{array}{c}\text { Sent \& Received, } O(n) \\
+ \text { [events \& msgs log } \\
\text { in slices } \\
\text { only during 3-phase] }\end{array}$ \\
\hline $\begin{array}{l}\text { Persistent } \\
\text { data structures }\end{array}$ & vector clocks & $\begin{array}{l}\text { vector clocks } \\
\text { or counters }\end{array}$ & - & counters \\
\hline $\begin{array}{l}\text { Entire history } \\
\text { used }\end{array}$ & yes & yes & no & no \\
\hline $\begin{array}{l}\text { Application } \\
\text { msg. overhead }\end{array}$ & vector clock, $O(n)$ & vector clock, $O(n)$ & - & - \\
\hline Processing & by initiator & by initiator & by initiator & by initiator \\
\hline Channels & FIFO & non-FIFO & non-FIFO & FIFO \\
\hline \# control msgs. & $(n-1)$ & $(n-1)$ & $6(n-1)$ & $6(n-1)$ \\
\hline
\end{tabular}

The messages and events in the logs are only the relevant events in all cases. The Sent and Received arrays and the notion of slice are explained in Section 3.

- Helary et al. [8] proposed an algorithm to detect a "fairly general" class of stable properties without characterizing this class any further. Informally, it uses local control variables at each process to track 1 ) whether a process is active or passive, 2) whether each incident channel is empty, and 3) whether each channel is closed, i.e., whether or not a message will ever be received on it. Whenever these variables change value so as to suggest the stable property might be true, the process sends the information (along with counts of the number of messages sent to and received from all processes) to the monitor process. The monitor process pieces together a global state using such information from all processes and if the stable predicate is true, it gets detected. The class of stable properties that can be detected is not characterized in [8], but they give examples of deadlock detection and termination detection. It appears that the algorithm can detect only those properties that can be expressed in terms of the quiescence of channels, i.e., variables that can track items 1, 2, and 3 above.

Neither Marzullo and Sabel [17] nor Schiper and Sandoz [21] showed any relationship between the classes of strong stable and locally stable predicates. Schiper and Sandoz conjectured that they are similar.

A strongly stable predicate differs from a strong stable predicate in that the predicate is evaluated over any cut, consistent or not, and variables at processes not in the consistent projection are also considered in evaluating the predicate. The predicate $\phi={ }_{\text {def }} x_{1}+x_{2}+\ldots+x_{n} \geq k$, where each $x_{i}$ is a monotonically nondecreasing variable at process $P_{i}$, is strongly stable but is not strong stable. Deadlock is strong stable but not strongly stable. The above strongly stable predicate $\phi$ is not locally stable.

The above surveyed algorithms can only detect some subclass of stable predicates-for example, they cannot detect "there are at most $k$ tokens" in a system where tokens can be lost and initially there are more than $k$ tokens. Furthermore, some of these algorithms [14], [17], [21] modify all application messages with vector timestamps and use the $\log$ of the message history to report to the algorithm. Hence, they do not meet all the assumptions P1P4 above.

\subsection{Main Idea}

The algorithm proposed in this paper can be used to detect any stable predicate and satisfies properties P1-P4 above. The proposed algorithm requires $6(n-1)$ control messages, where $n$ is the number of processes in the system. A simple and elegant three-phase strategy of uncoordinated observation of local states is used to give a consistent snapshot from which any stable property can be detected. Minimal data structures are used only during the execution of the three phases; the executions between the first and second phases, and between the second and third phases, are termed as slices. Two versions of the algorithm are presented:

Version 1. The first version records consistent process states without requiring the channels to be FIFO.

Version 2. The second version records process states and channel states consistently but requires FIFO channels. (Two variants of this version of the algorithm are also presented in a later section.)

Table 1 compares the features and the complexities of the proposed algorithm with those of the algorithms in [17], [21].

Stable predicate detection is a special case of global state detection. Our stable predicate detection algorithms follow directly from the algorithms we propose for recording a distributed snapshot efficiently. Since the seminal elegant algorithm of Chandy and Lamport [4], which is a noninhibitory algorithm that requires FIFO channels and $2 l$ messages for recording the snapshot, plus additional messages for assembling the snapshot, several other algorithms have been proposed. A good survey and comparison of such algorithms is given in [11]. In our approach, a simple and elegant three-phase strategy of uncoordinated observation of local states gives a consistent distributed snapshot. The first version of the snapshot algorithm (v.1) records consistent process states without requiring FIFO channels and without using any form of 
TABLE 2

Features of Global Snapshot Algorithms

\begin{tabular}{|l|c|c|c|c|c|c|}
\hline Algorithm & Channels & Inhibitory & $\begin{array}{c}\text { Application } \\
\text { messages } \\
\text { modified }\end{array}$ & $\begin{array}{c}\text { Number of } \\
\text { control } \\
\text { messages }\end{array}$ & $\begin{array}{c}\text { Snapshot } \\
\text { collection } \\
\text { needed }\end{array}$ & $\begin{array}{c}\text { Message } \\
\text { history } \\
\text { used }\end{array}$ \\
\hline $\begin{array}{l}\text { Chandy-Lamport [4] } \\
\text { Sridhar-Sivilotti [23] }\end{array}$ & FIFO & $\mathrm{N}$ & $\mathrm{N}$ & $O\left(n^{2}\right)$ & $\mathrm{Y}$ & $\mathrm{N}$ \\
\hline Spezialetti-Kearns [22] & FIFO & $\mathrm{N}$ & $\mathrm{N}$ & $O\left(n^{2}\right)$ & $\mathrm{Y}$ & $\mathrm{N}$ \\
\hline Venkatesan [25] & FIFO & $\mathrm{N}$ & $\mathrm{N}$ & $O\left(n^{2}\right)$ & $\mathrm{Y}$ & $\mathrm{N}$ \\
\hline Helary [7] (wave sync.) & FIFO & $\mathrm{Y}$ & $\mathrm{N}$ & $O\left(n^{2}\right)$ & $\mathrm{Y}$ & $\mathrm{N}$ \\
\hline \hline Helary [7] (wave sync.) & non-FIFO & $\mathrm{Y}$ & $\mathrm{N}$ & $O\left(n^{2}\right)$ & $\mathrm{Y}$ & $\mathrm{N}$ \\
\hline Lai-Yang [14] & non-FIFO & $\mathrm{N}$ & $\mathrm{Y}(\mathrm{p} . \mathrm{b})$. & $O\left(n^{2}\right)$ & $\mathrm{Y}$ & $\mathrm{Y}$ \\
\hline LRV [16] & non-FIFO & $\mathrm{N}$ & $\mathrm{Y}(\mathrm{p} . \mathrm{b})$. & $O\left(n^{2}\right)$ & $\mathrm{Y}$ & $\mathrm{Y}$ \\
\hline Mattern [19] & non-FIFO & $\mathrm{N}$ & $\mathrm{Y}(\mathrm{p} . \mathrm{b})$. & $O(n)$ & $\mathrm{Y}$ & $\mathrm{N}$ \\
\hline \hline Acharya-Badrinath [1] & $\mathrm{CO}$ & $\mathrm{N}$ & $\mathrm{N}$ & $2 n$ & $\mathrm{~N}$ & $\mathrm{~N}$ \\
\hline Alagar-Venkatesan [2] & $\mathrm{CO}$ & $\mathrm{N}$ & $\mathrm{N}$ & $3 n$ & $\mathrm{~N}$ & $\mathrm{~N}$ \\
\hline \hline $\begin{array}{l}\text { Proposed snapshot v.1 } \\
\text { (w/o channel states) }\end{array}$ & non-FIFO & $\mathrm{N}$ & $\mathrm{N}$ & $6 n$ & $\mathrm{~N}$ & $\mathrm{~N}$ \\
\hline Proposed snapshot v.2 & FIFO & $\mathrm{N}$ & $\mathrm{N}$ & $6 n$ & $\mathrm{~N}$ & $\mathrm{~N}$ \\
\hline
\end{tabular}

The acronym p.b. denotes that control information is piggybacked on the application messages.

message send/receive or event counters. The second version (v.2) records process states and channel states consistently but requires FIFO channels. Critchlow and Taylor have shown that, for a system with non-FIFO channels, a snapshot algorithm must either use piggybacking or use inhibition [5]. Hence, the second version of the algorithm cannot be improved upon to also record channel states while retaining the properties of no inhibition and no piggybacking while using non-FIFO channels.

We compare the properties of the proposed snapshot algorithm (both versions) with the properties of the existing algorithms in Table 2. Existing algorithms can be classified into three broad classes:

- The baseline Chandy-Lamport algorithm [4], and its variants, the Spezialetti-Kearns algorithm [22], Venkatesan's algorithm [25], the Sridhar-Sivilotti algorithm [23], and Helary's wave synchronization algorithm [7] assume FIFO channels, are noninhibitory, do not modify messages, and require $O\left(n^{2}\right)$ control messages to record the snapshot. Additional messages are required to collect the locally recorded snapshot views.

- For non-FIFO channels, Helary's inhibitory algorithm [7], the Lai-Yang algorithm [14], the LiRadhakrishnan-Venkatesh (LRV) algorithm [16], and Mattern's termination-detection-based algorithm [19] have been proposed. All these (except Helary [7], which is inhibitory) require application messages to be modified. Additional messages are required to collect the locally recorded snapshots. These algorithms (except Mattern's [19], which uses termination detection, and Helary's [7], which uses inhibition) require entire message histories to be recorded and piggybacked as part of the snapshot recording.

- Algorithms such as those by Acharya and Badrinath [1] and Alagar and Venkatesan [2] assume causally ordered (CO) message delivery and a single initiator and require $O(n)$ control messages. They do not require additional messages to explicitly collect the locally recorded snapshots because the snapshot gets assembled at the initiator as part of the algorithm. However, causally ordered channels are expensive. They require $O\left(n^{2}\right)$ overhead and delivery of messages may have to be delayed.

Other variants that require the use of vector timestamps [6], [18] are not considered here because of their high cost. As related work, Helary and Raynal gave a broad framework [9] based on iterative guarded wave sequences, which suggests a way to derive algorithms for specific predicates.

\subsection{Summary of Main Contributions}

1. As seen from Table 2, the snapshot algorithm we propose for FIFO channels is linear in the number of messages, (P1) does not modify the application messages, (P2) is noninhibitory, (P3) does not require the message history, and (P4) allows any process to initiate the algorithm. This is an important contribution to a fundamental problem.

2. The non-FIFO version of our snapshot algorithm can be used to detect locally stable predicates, under assumptions P1 through to P4.

3. The FIFO version of our snapshot algorithm can be used to detect any stable predicate, assuming P1 through P4. This algorithm is compared with other stable predicate detection algorithms in Table 1.

Outline. Section 2 gives the system model. Section 3 presents the proposed 3-phase snapshot algorithm (version 1) that works with non-FIFO channels but does not record channel states. Section 4 shows how to adapt this algorithm to record channel states if FIFO channels are assumed (version 2). Section 4 gives the complexity analysis. Section 5 shows how the respective algorithms can be used to detect any stable predicate (or a locally stable predicate) under the assumptions P1 through P4. Section 7 discusses additional variants of the proposed algorithms. Section 8 gives the conclusions.

\section{System Model}

The system model of the asynchronous reliable distributed system is as follows: We assume all messages are delivered 


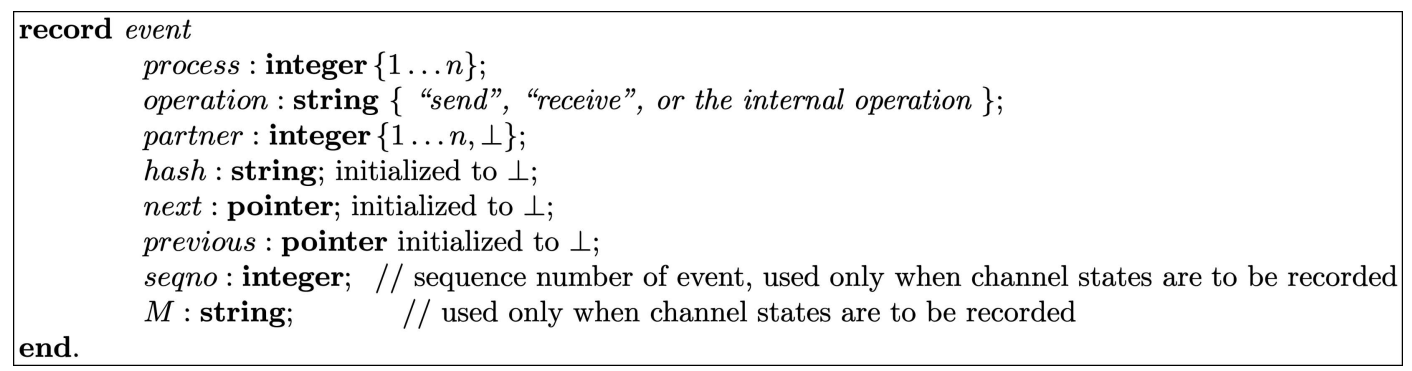

Fig. 1. Data structure of the event record. The event sequence is a doubly linked list.

in finite time. An asynchronous execution in a distributed system with $n$ processes is modeled as an execution $(E, \prec)$, where $\prec$ is the causality relation on the set of events $E$ in the execution. $E=\bigcup_{i \in N} E_{i}$, where $E_{i}$ is the totally ordered chain of events at process $P_{i}$. An event $e$ executed by $P_{i}$ is denoted $e_{i}$. The potential causality or the "happens before" relation $\prec$ on $E$ is the transitive closure of the local ordering relation on each $E_{i}$ and the ordering imposed by message send events and message receive events [15]. The execution history at a process $P_{i}$ is a sequence

$$
\left\langle s_{i}^{0}, e_{i}^{1}, s_{i}^{1}, e_{i}^{2}, s_{i}^{2}, e_{i}^{3}, s_{i}^{3}, \ldots\right\rangle
$$

of alternating states and local events, where $s_{i}^{0}$ is the initial state of process $P_{i}$ and event $e_{i}^{x}$ causes a state transition from state $s_{i}^{x-1}$ to state $s_{i}^{x}$. Due to the bijective mapping among the noninitial states and the events at a process, reference to an event implicitly identifies the corresponding state.

A cut $C$ is a subset of $E$ such that if $e_{i} \in C$, then

$$
\left(\forall e_{i}^{\prime}\right) e_{i}^{\prime} \prec e_{i} \Longrightarrow e_{i}^{\prime} \in C .
$$

In contrast, a consistent cut $C$ is a subset of $E$ such that if $e \in C$ then $\left(\forall e^{\prime}\right) e^{\prime} \prec e \Longrightarrow e^{\prime} \in C$. The system state after the events in a cut is a global state; if the cut is consistent, the corresponding system state is a consistent global state and denotes a meaningful observation of a global state [4]. Formally, a global snapshot or global state $G S$ consists of

- a set of local states $\left\{s_{1}^{x_{1}}, s_{2}^{x_{2}} \ldots s_{n}^{x_{n}}\right\}$ and

- a set of the states of channels $C_{i, j}(\forall i \forall j)$, where $\operatorname{state}\left(C_{i j}\right)=\operatorname{transit}\left(e_{i}^{x_{i}}, e_{j}^{x_{j}}\right)$, the set of messages sent by $P_{i}$ up to event $e_{i}^{x_{i}}$ and not received by $P_{j}$ up to event $e_{j}^{x_{j}}$.

An execution slice is defined as the difference of two cuts $D \backslash C$, where $C \subseteq D$. This definition of a slice is somewhat different from the traditional definition in software engineering [26]. The slice is also referred to as a suffix of $C$ with respect to $D$. The default value of $C$ is $\emptyset$, in which case the slice is the cut $D$. Observe that each cut $D$ is also a slice (i.e., $D \backslash \emptyset$ ). A slice $D$ which is a set has a natural representation as an array $D[1 \ldots n]$ of sequences of events, one sequence per process. This representation captures the local order of events at each process without additional overhead. The reference to a slice-to its definition as a set or to its representation as an array of sequence of eventsshould be clear from context. The suffix and prefix of a slice $D[1 \ldots n]$ have a natural interpretation, namely, as the suffix or prefix of each $D[i], 1 \leq i \leq n$.
A stable predicate is a predicate that remains true once it becomes true [4]. Let $\models$ denote the satisfaction operator for a formula. Formally, if predicate $\phi$ is stable, then

$$
C \models \phi \text { implies that }(\forall D \mid D \supseteq C) D \models \phi .
$$

Events and messages of the snapshot algorithm form a superimposed control execution that does not affect the application execution $E$ or the partial order $(E, \prec)$ which is defined only on the application events. Among the application events and messages, the events and messages that are relevant to the predicate of interest are termed as relevant events and messages, respectively. We assume that all events, variables, and messages recorded in the algorithms are only the relevant ones. For a send or receive event that occurs at process indexed $i$, unless otherwise specified, we use $\tilde{i}$ to denote index of the process at which the corresponding receive or send event occurs.

\section{Three-Phase Algorithm: Local States, NON-FIFO CHANNELS}

\subsection{Strategy}

The snapshot algorithm was inspired by the two-phase deadlock detection algorithm [10], [12]. The algorithm has three serially executed phases, in each of which uncoordinated "snapshots" that may be inconsistent are recorded. A consistent global state that lies between the first and the second inconsistent "snapshots" is computed with the help of the third "snapshot." Any process can initiate the algorithm, but it has to do some local processing. Each phase involves the initiator sending a request to each other process, and then the processes replying to the initiator. The initiator can communicate directly to/from the various processes, or a wave algorithm [20] can be used in conjunction with a superimposed topology such as a ring or a tree. The snapshot algorithm is independent of this detail.

Phase I. The initiator requests the processes to begin recording an execution slice. Each process replies to the initiator when it receives the Phase I request, by piggybacking the local state on this reply. Each process then begins to record a slice as a sequence of (relevant) events, where each event has the data structure shown in Fig. 1. Specifically,

- For send and receive events, the hash of the message (such as SHA-1) and the corresponding receiver/ sender are also recorded in fields hash and partner, respectively. 


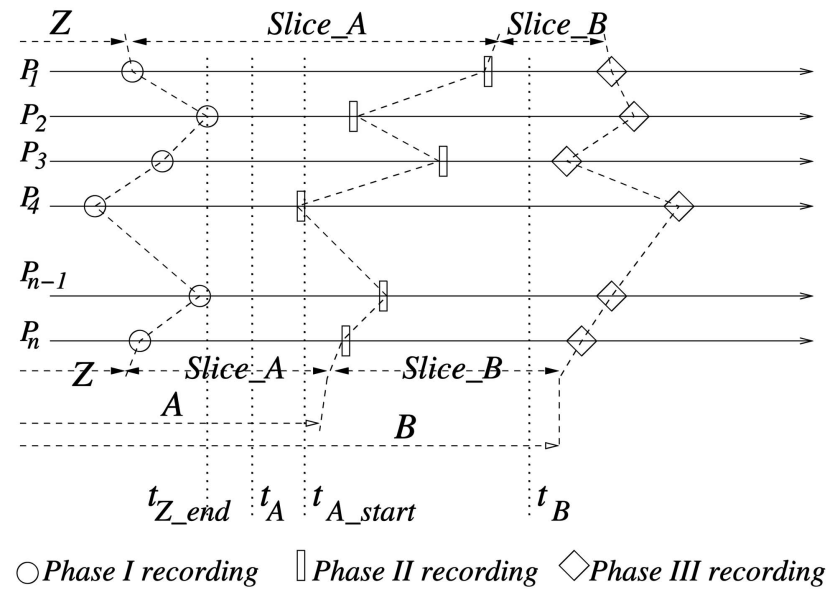

Fig. 2. Timing diagram to illustrate three-phase uncoordinated recording of a global snapshot. The initiator could be any of the processes.

- The field seqno gives the sequence number of the event at its process. The field $M$ records the message received. These fields are used only if channel states are to be recorded (Version 2 of the algorithm).

The cut after which processes begin recording the slice is denoted $Z$. The state after this cut is represented by the array State $Z[1 \ldots n]$ at the initiator. The local states in State_ $Z$ may represent an inconsistent global state due to the uncoordinated nature of their recording.

Phase II. The initiator waits for the Phase I replies from all other processes. It then sends a Phase II request to all the processes. When a process receives a Phase II request, it reports the slice of its execution since the time it began recording its slice in Phase I until the time it chooses to reply to the Phase II request. The local slice of each process that is reported to the initiator is stored by the initiator in array Slice_$A[1 \ldots n]$. The cut containing all the events up to the local time at which each process replies to the Phase II request is denoted as $A$. After sending the Phase II reply, each process starts recording the next slice.

Phase III. The initiator waits to receive the Phase II reply from each process. It then sends a Phase III request to all the processes. When a process receives a Phase III request, it reports the slice of its execution that was recorded since the time it replied to the Phase II request until the time it chooses to reply to the Phase III request. The local slice of each process that is reported to the initiator is stored by the initiator in array Slice_$_{-} B[1 \ldots n]$. The cut containing all the events up to the local time at which each process replies to the Phase III request is denoted as $B$.

The possibly inconsistent values that are reported to the initiator in $S_{t a t e} Z$, Slice_A, and $S_{\text {Slice_}} B$ are illustrated in Fig. 2. The initiator constructs a consistent global cut $S$, such that $Z \subseteq S \subseteq A$ using Slice $_{-} A$ and Slice_B $_{\text {B }}$. In Slice_A, there are six types of send and receive events (see Fig. 3).

1. send event, for a message that gets delivered in $Z$,

2. send event, for a message that gets delivered in Slice_A,

3. send event, for a message that gets delivered after Slice_A,

4. receive event, for a message that was sent in $Z$,

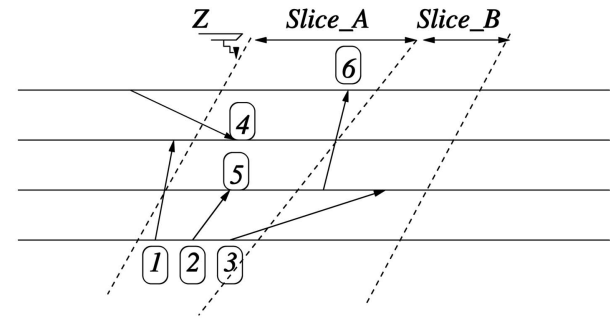

Fig. 3. Timing diagram to illustrate six types of events in Slice_$_{-}$.

5. receive event, for a message that was sent in Slice_$_{-} A$, and

6. receive event, for a message that was sent after Slice_A. Due to the existence of time instant $t_{B}$ as shown in Fig. 2, the message must have been sent in Slice $_{B}$.

This suggests two approaches to construct a consistent cut $S$ :

Extend $Z$ : Extend $Z$ to a consistent cut $S$ by adding that prefix from Slice_$_{-} A$ satisfying the following:

- The prefix contains all events of type 1 and no events of type 6 , and

- $Z \cup\{$ events in the prefix $\}$ is a consistent cut.

Reduce $A$ : As cut $A$ may not be a consistent cut, we can construct a consistent cut $S$ based on $A$ by subtracting the suffix that contains

- all events of type 6 and

- additional events to ensure that $A \backslash\{$ events in suffix $\}$ is a consistent cut.

Observe that in real time, all events of type 1 precede all events of type 6. Define $t_{A_{-} \text {start }}, t_{Z_{-} \text {end }}$, and $t_{A}$ (see Fig. 2) as follows:

- Let $t_{A_{-} \text {start }}$ denote the time instant of the first local recording of Phase II among all the processes.

- Let $t_{Z_{-} \text {end }}$ denote the time instant of the last local recording of Phase I among all the processes.

- Let $t_{A}$ be any instant in global time such that $t_{Z_{-} \text {end }}<t_{A}<t_{A_{-} \text {start }}$.

Let $S(t)$ be the cut at global time $t$. The cut $S\left(t_{A}\right)$ which is a consistent cut satisfying the conditions on cut $S$ above (for both the "Extend $Z$ " and "Reduce $A$ " approaches) must exist.

In the exposition of our algorithm (Version 2), we follow the "Reduce $A$ " approach. In Section 7, we give two variants of Version 2 of the algorithm:

Version 2A. This variant leverages the use of the data structures more efficiently than Version 2.

Version 2B. This variant uses the "Extend $Z$ " approach.

We now define the cut $S_{\max }$.

- $S_{\max }=Z \bigcup\{$ events in the largest prefix from Slice_A that does not include an event of type 6$\}$.

See Fig. 4 . Note that $S_{\max }$ may not be consistent. The cut $S_{\max }$ includes $S\left(t_{A_{-} \text {start }}\right)$ and $A$ includes $S_{\max }$.

The algorithm constructs the largest consistent cut $S$ such that $S\left(t_{A_{-} \text {start }}\right) \subseteq S \subseteq S_{\max } \subseteq A$, in two steps: 


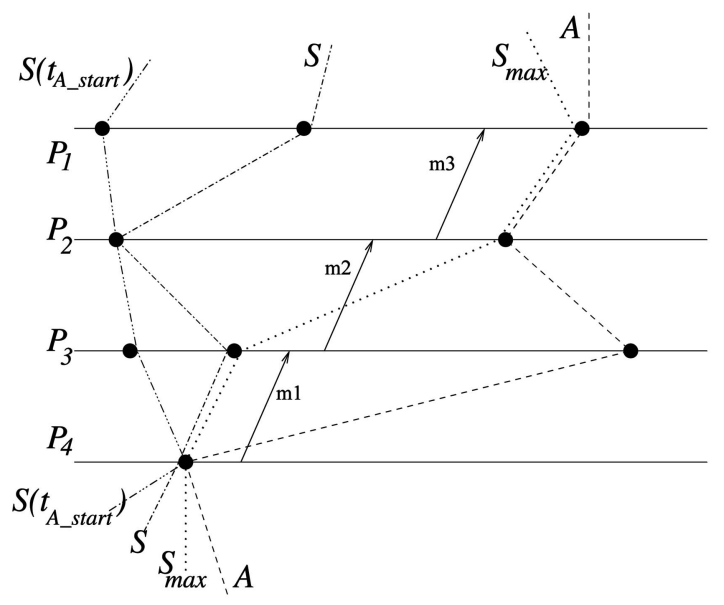

Fig. 4. Timing diagram to illustrate example relationships among cuts $A$, $S_{\text {max }}, S$, and $S\left(t_{A_{-} s t a r t}\right)$. For each cut, only the latest event at each process is shown.

1. Slice_B helps to construct $S_{\max }$ by identifying messages sent in Slice_B that were received in Slice_A. For example, in Fig. 4, the message $m 1$ has its send event in $S_{\text {Slice_B }}$. The minimum slice suffix from $S_{\text {Slice_}} A[i](\forall i)$ that contains the receive event of each message sent in Slice_$_{-}$is removed to construct $S_{\max }$. In the example, $S_{\max }$ is therefore different from A. $S_{\max }$ may still be inconsistent due to receive events for messages that were sent from Slice_A. In the example, the message $m 2$ causes $S_{\max }$ to be inconsistent.
2. The algorithm then removes the minimum slice suffix from $S_{\max }$ to remove receive events that cause inconsistency. This is done iteratively to construct a consistent cut $S$. In the example, messages such as $m 2$ and then $m 3$ need to be removed.

The set of all cuts forms a lattice [18]. As $S_{\max }$ is defined to be the cut that is the largest subset of $A$ and does not contain any receive events for which messages were sent in Slice_B, $S_{\max }$ is unique due to the lattice property. The set of all consistent cuts also forms a lattice. As $S$ is defined to be the largest consistent cut within $S_{\text {max }}, S$ is also unique. As $A, S_{\text {max }}$, and $S$ are all uniquely defined, the minimum slice suffixes to be removed to construct $S_{\max }$ and $S$ are also well-defined.

\subsection{Three-Phase Algorithm}

Fig. 5 gives the code for the three-phase processing. Step 1 describes the processing at the initiator. Step 2 describes the processing at all the nodes. When Slice_$_{-} A$ and Slice $_{-} B$ are recorded, note the following:

- Messages are not modified with sequence numbers to conform to P1. In addition, no counters for sequence numbers for the messages sent or received, or for the event count, are used at processes. A hash or checksum computed on each message sent or received is stored in the log of the slice, to enable matching a message in the sender's $\log$ with the same message in the receiver's log. By choosing an appropriate hash function, the probability of collisions of the hash values can be made arbitrarily small.

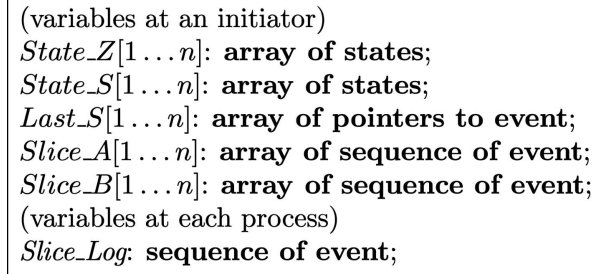

// Phase 1 state recording, stored at initiator // states in the constructed consistent cut // pointers to last events in constructed consistent cut // Phase 2 slice recordings, stored at initiator // Phase 3 slice recordings, stored at initiator // log of local events within a slice

(2) Process $P_{j}$ executes the following, for each $j$ such that $1 \leq j \leq n$.

(2a) On receiving Request_Phase_1_Report from $P_{\text {init }}$,

(2b) send Phase_1_Report(state ${ }_{j}$ ) to $P_{\text {init }}$;

(2c) Begin recording sequence of events in Slice_Log;

(2d) On receiving Request_Phase_2_Report from $P_{\text {init }}$,

(2e) send Phase_2_Report(Slice_Log) to $P_{\text {init }}$;

(2f) Reset Slice_Log; begin recording sequence of events in Slice_Log;

$(2 \mathrm{~g})$ On receiving Request_Phase_3_Report from $P_{\text {init }}$,

(2h) send Phase_3_Report(Slice_Log) to $P_{\text {init }}$;

(2i) Stop recording events in Slice_Log. 


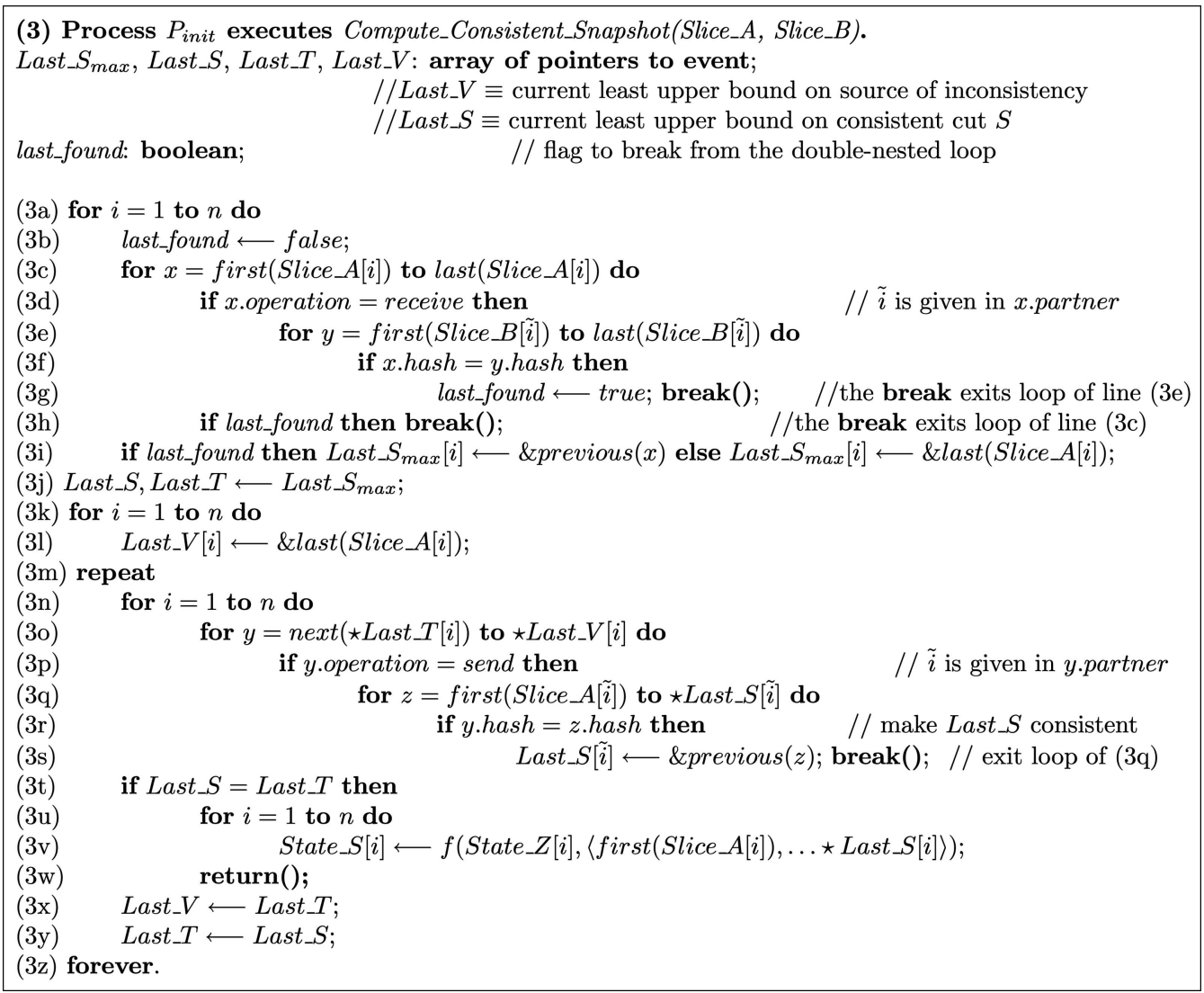

Fig. 6. Iteratively constructing a consistent cut.

After $S_{\text {Slice }} A$ and $S l i c e \_B$ have been reported to $P_{\text {init }}$ at the end of Phases II and III, the initiator invokes procedure Compute_Consistent_Snapshot given in Fig. 6 to construct a consistent cut $S$. The algorithm uses two temporary cuts $T$ and $V$. Cuts $S_{\max }, S, T$, and $V$ are not explicitly stored as sets but are implicitly identified by vectors Last_S $S_{\max }$, Last_S, Last_T, and Last_V of pointer variables. The $i$ th element (where $1 \leq i \leq n$ ) of any of these vectors points to the last event at process $P_{i}$ in the corresponding cut.

Slice_A $A[i]$ and Slice_B $[i]$, which are sequences of events, are represented as doubly linked lists of type event (see Fig. 7). The first event is preceded by a head and the last

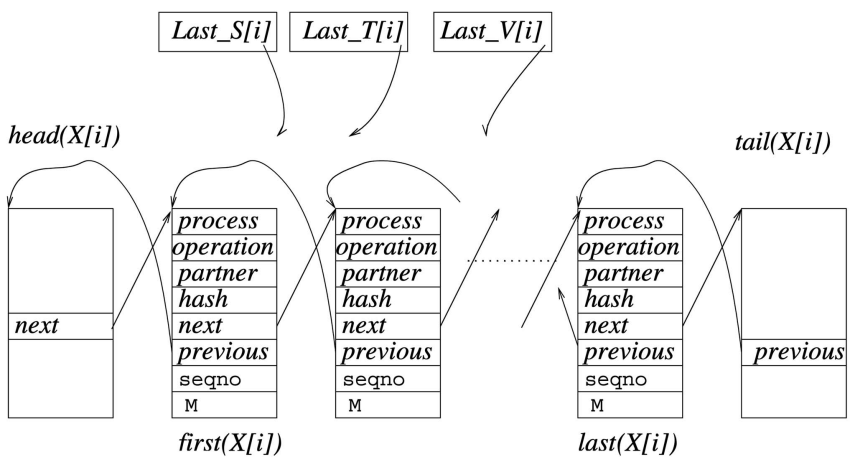

Fig. 7. Implementation of event sequence $X[i]$ (e.g., Slice_A[i] and Slice_B $B[i])$ as a doubly linked list. The fields in the typewriter font are present only if channel states are to be recorded. event is succeeded by a tail. The operations first, last, next, and previous with the standard semantics on these lists are assumed. The vectors Last_S $S_{\max }$, Last_S, Last_T, and Last_V of pointer variables point within the doubly linked lists of Slice_A. See Fig. 7.

In Fig. 6, lines 3a through 3i construct $S_{\max }$ by removing the minimum suffix from Slice_ $A$, in which messages from Slice $B$ are received. The solution cut $S$ is then constructed by iteratively removing the minimum slice suffix from cut $S_{\max }$ to get a consistent cut. Line $3 \mathrm{j}$ initializes the array of pointer variables Last_S and Last_T to Last_S $S_{\max }$.

- Array Last_S is always set to the best known least upper bound of the consistent cut $S$ that is sought.

- Array Last_V denotes the best known least upper bound on the cut $V$ such that only messages sent in the slice $V \backslash S$ may cause $S$ to be inconsistent. Last_V $[i]$ (for $1 \leq i \leq n$ ) is initialized so as to point to the last event in Slice_A (lines $3 \mathrm{k}$ and 31).

- Array Last_T is a temporary variable that is used to update $L a s t_{-} V$ at the end of an iteration to the value held by Last_S at the start of that iteration.

The goal is to construct $S$ such that $S$ is consistent.

The main repeat loop (lines $3 \mathrm{~m}$ to $3 \mathrm{z}$ ) updates $S$ and $V$ iteratively. In each iteration, it examines each event $y$ in the slice $V \backslash S$ to determine if a message sent at that event was received at an event $z$ that belongs to slice $S$ (lines $3 n$ to $3 r$ ). A message at the sender is matched with the same message at the receiver by comparing their hashes/checksums 


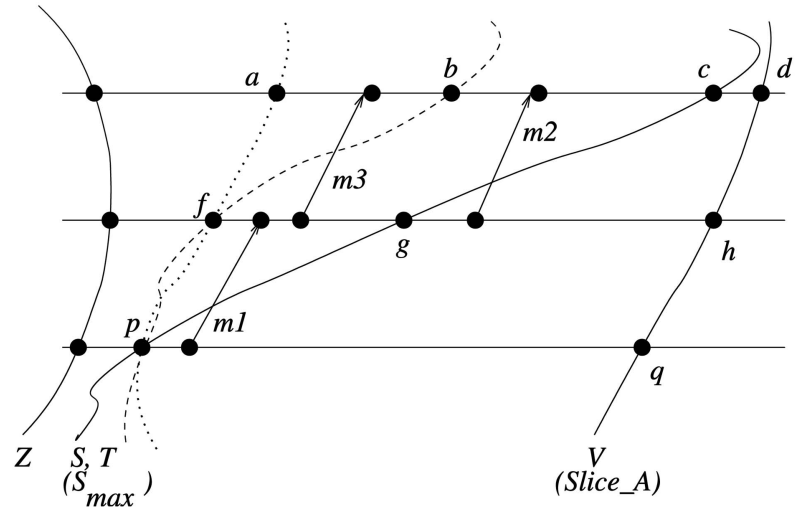

Fig. 8. Example showing removal of inconsistencies.

(line 3r). When such an event $z$ at some process $P_{\tilde{i}}$ is identified, the algorithm updates slice $S$ by deleting the suffix at $P_{\tilde{i}}$ from event $z$ onward (line 3s). This removes the inconsistency in slice $S$ caused by the message from $y$ to $z$.

Toward the end of one iteration of the repeat loop, $V$ is updated to $T$ (line 3x), and then $T$ is updated to $S$ (line 3y) for the next iteration. Observe that $S$ may still be inconsistent because a message sent in $V \backslash S$ may be received in $S$. Hence, the repeat loop must be executed again unless $S=T$ (line 3t), i.e., no inconsistent-causing message from $V \backslash S$ to $S$ was found in the current iteration. The global state State_S is then computed as shown in lines $3 \mathrm{t}$ to $3 \mathrm{v}$. The function $f$ begins with event first(Slice_A $A[i])$ for all $i$, and reruns the computation slice

$$
\langle\text { first }(\text { Slice_A } A[i]) \ldots \star \text { Last_S}[i])\rangle
$$

from $S_{\text {tate }} Z[i]$ to construct State $_{-} S$. The rerun of the partial slice is achieved by applying the transformations in the operation fields of noncommunication events in the sequence of events above.

In the code, if the previous operation on an event in line $3 \mathrm{i}$ points to the head of the sequence, then

- in line 3o, next $\left(\star\right.$ Last_T $\left._{-}[i]\right)$ points to the first event in that sequence, if any, or else to the tail, and

- $\quad$ in line $3 q$, Last_S $[i]$ points to the head of that same sequence and the loop does not execute.

Similarly, in line $3 \mathrm{~s}$, if previous $(z)$ points to

$$
\operatorname{head}\left(\text { Slice_}_{A}[j]\right) \text {, }
$$

then, in line $3 \mathrm{v}, \star$ Slice_$\_S[j]$ is the head of a sequence and State_ $S[j]$ is simply set to State_Z $[j]$.

Example. In Fig. 8, assume the events pointed to by Last_S, Last_T are $[c, g, p]$, as shown by regular curves. Assume that the events pointed to by Last_V are $[d, h, q]$. The initial values of Last_S, Last_T and of Last_V are shown in parentheses.

Iteration 1 of repeat loop. After line 3s, $\star L a s t_{-} S=$ $[b, f, p], \star L_{\text {Last_}} T=[c, g, p]$, and $\star L a s t_{-} V=[d, h, q]$. After line $3 \mathrm{y}, \star L a s t_{-} S, \star$ Last_T$_{-}=[b, f, p]$, and $\star L a s t_{-} V=[c, g, p]$.

Iteration 2 of repeat loop. After line $3 \mathrm{~s}, \star$ Last_S $=$ $[a, f, p], \star$ Last_T$_{-}=[b, f, p]$, and $\star L a s t_{-} V=[c, g, p]$. After line $3 \mathrm{y}, \star$ Last_S, $\star$ Last_T $=[a, f, p]$, and $\star$ Last_V$=$ $[b, f, p]$.
Iteration 3 of repeat loop. In line $3 \mathrm{t}, \star$ Last_$_{-} S=$ $\star$ Last_T $=[a, f, p]$. So, there is no inconsistency in $S$ and the algorithm exits from the loop.

The correctness of the recorded global state is based on the following theorems proved in the Appendix.

Lemma 1. In each iteration of the repeat loop of lines $3 m$ to $3 z$ of Compute_Consistent_Snapshot in Fig. 6, only the minimum suffix from the cut identified by Last_S that is needed to remove inconsistency-causing messages is deleted.

Lemma 2. For any inconsistency-causing chain $M$ given by $\left\langle m^{1}, m^{2}, \ldots m^{k}\right\rangle$, after $j$ iterations of the repeat loop in lines $3 m$ to $3 z$ in Fig. 6, the chain becomes $M^{\prime}$ given by $\left\langle m^{j+1} \ldots m^{k}\right\rangle$, where $s^{j+1} \notin$ Last_S.

Lemma 3. The longest inconsistency-causing chain has length $n-1$.

Theorem 1 (Termination). Procedure Compute_Consistent_ Snapshot in Fig. 6 terminates in $n$ iterations of the repeat loop of lines $3 m-3 z$.

Theorem 2 (Correctness of Local Recordings). The algorithm initiated by $P_{\text {init }}$ terminates by finding a consistent cut $S$, where $S\left(t_{A_{-} \text {start }}\right) \subseteq S \subseteq S_{\max }$.

\section{Consistent State and Channel Recording UNDER FIFO CHANNELS}

This section presents an enhanced algorithm that also records channel states if channels are FIFO. This algorithm can be used to detect any stable predicate. In the previous algorithm, Slice_B was used to construct Last_S $S_{\max }$ by identifying those messages received in Slice_A that were sent in Slice $_{-} B$. Here, Slice_$B$ is also used to capture the channel states. To do so, we require that the recording within Slice_B completes at each process when the messages sent by other processes to that process in (and before) Slice_ $A$ have been received. This condition is detectable using some extra control information distributively sent to the initiator in the Phase II reply messages and then conveyed on the Phase III request received from the initiator.

Fig. 9 shows the three-phase processing. The underlined pseudocode and data structures mark the differences from the algorithm in Fig. 5. Procedure Compute_Consistent_ Snapshot in Fig. 6 constructs a consistent cut from Slice_ $\bar{A}$ and Slice_B $_{3}$, as described in Section 3.

The state of channel $C_{i, j}$ after events $\star L a s t \_S[i]$ and $\star L a s t_{-} S[j]$, is defined as the set of messages sent by $P_{i}$ up to event $\star L a s t_{-} S[i]$ that are not received until event $\star L_{a s t} S[j]$ at $P_{j}$ (and hence received later in Slice_A and Slice_B). Procedure Compute_In_Transit_Messages in Fig. 10 computes the channel states transit $\left(\star\right.$ Last_$_{-} S[i], \star$ Last_S$\left._{-}[j]\right)$, for all $\langle i, j\rangle$ pairs, using

- integer arrays Global_Sent $[1 \ldots n, 1 \ldots n]$ and

$$
\text { Global_Received }[1 \ldots n, 1 \ldots n]
$$

at the initiator during the processing of the algorithm, and

- integer vectors Received $[1 \ldots n], \operatorname{Sent}[1 \ldots n]$, and

$$
\text { Must_Receive }[1 \ldots n]
$$




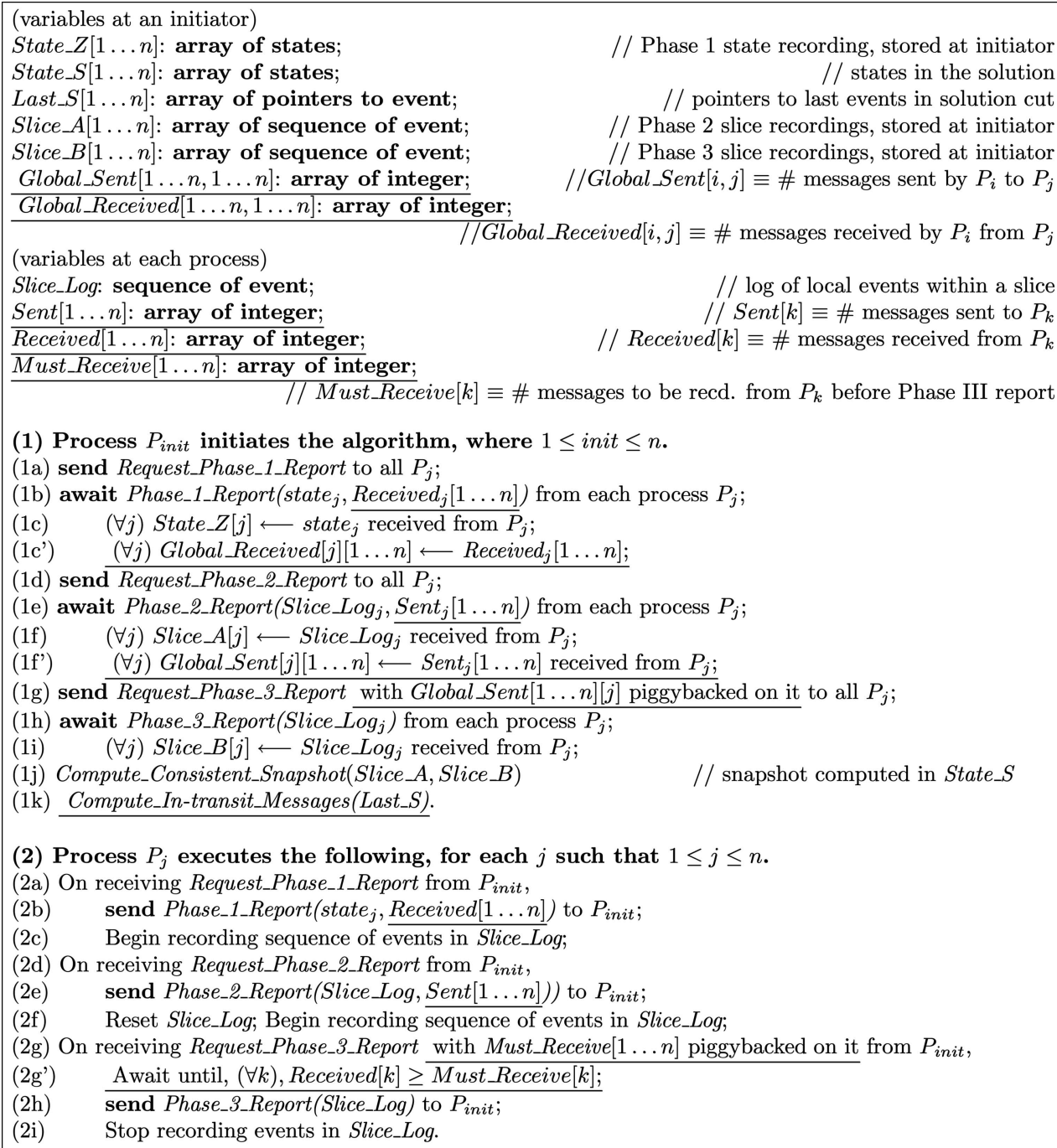

Fig. 9. Three-phase algorithm to record a global snapshot and channel states. Continued in Fig. 10.

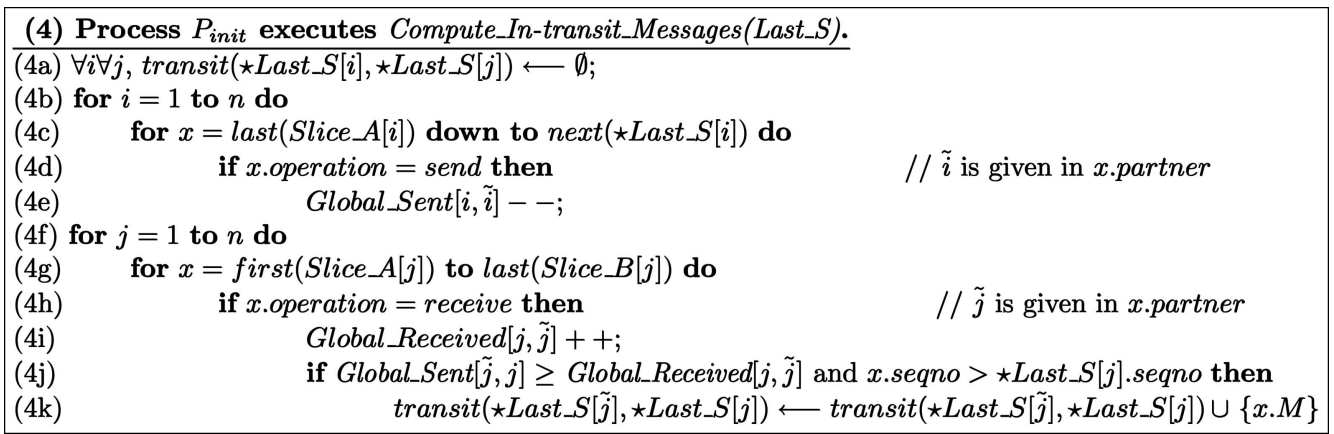

Fig. 10. Computing in-transit messages.

at each node. Sent $[j]$ and Received $[j]$ track the number of messages sent to and received from process $P_{j}$, respectively.

One important difference from the previous algorithm is that sequence numbers are used to count events at a process. This is because for a message received within Slice_B, it becomes necessary to identify whether the message was sent before $S_{\text {Slice_}} A$ or after Slice_$_{-} B$. To see this further, consider Fig. 11. Sequence numbers within each local execution can help to determine whether $m 1$ and $m 2$ were sent before or after the three-phase recordings. To compute the channel state while satisfying conditions P1 through P4 and, specifically, that no sequence numbers can be tagged on messages, three issues need to be addressed:

1. Messages sent by $P_{i}$ to $P_{j}$ before event $\star$ Last_S$S[i]$ must have reached $P_{j}$ by last $\left(\right.$ Slice $\left._{-} B[j]\right)$.

This is ensured by using the local Sent vector at each process and the Global_Sent array at the initiator. In the Phase II recording reported to the initiator, the Sent vectors reported (line 2e) are used to populate 


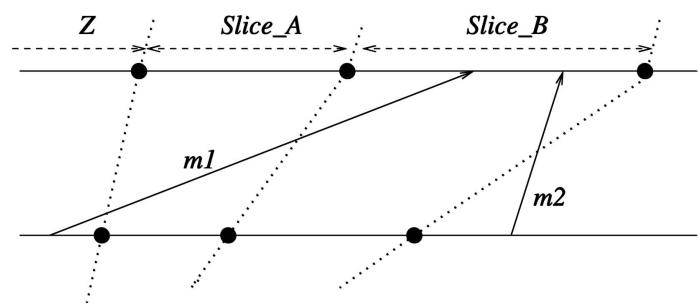

Fig. 11. Pre-Slice_ $A$ and post-Slice_B message send events need to be distinguished from each other.

Global_Sent (line $1 \mathrm{f}^{\prime}$ ). The Phase III request sent to each process contains the piggybacked information about how many messages have been sent to that process by other processes (line $1 \mathrm{~g}$ ). A process postpones the end of the recording of the local log of Slice_B until all these number of messages, remembered in array Must_Receive, have been delivered locally (line $2 \mathrm{~g}^{\prime}$ ).

2. The set of messages sent by $P_{i}$ to $P_{j}$ up to $\star L a s t \_S[i]$, denoted as $\mathcal{X}$, should be identifiable.

This set contains all the messages sent by $P_{i}$ (to $P_{j}$ ) with sequence numbers less than or equal to the value of $S e n t[j]$ after $\star$ Last_S $[i]$. This value is computed (lines 4b-4e) using Global_Sent and Slice_A that are reported in Phase II. The resulting message count is stored in situ in Global_Sent $[i, j]$.

3. The set of messages received by $P_{j}$ from $P_{i}$ after $\star L a s t \_S[j]$, denoted as $\mathcal{Y}$, should be identifiable.

The messages received by $P_{j}$ are enumerated in lines (4f-4k) using Global_Received, Slice_A, and Slice_B. The enumeration is done dynamically in situ in Global_Received $[j, i]$. Among these messages received by $P_{j}$, those that are received after $\star L a s t_{-} S[j]$ must be received at events $x$ satisfying the condition $x$.seqno $>\star$ Last_S $[j]$.seqno.

From the above and the definition of transit, we have

$$
\text { transit }(\star \text { Last_S } S[i], \star \text { Last_S }[j])=\mathcal{X} \cap \mathcal{Y}
$$

which is computed in line $4 \mathrm{k}$ :

$$
\begin{gathered}
\left\{M \text { received by } P_{j} \text { at event } x \mid(\text { Global_Received }[j, i] \text { at } x)\right. \\
\leq \text { Global_Sent }[i, j] \bigwedge x . \text { seqno }>\star \text { Last_S }[j] . \text { seqno }\}
\end{gathered}
$$

The correctness proof of the recorded channel states is based on the following theorem, whose proof is given in the Appendix.

Theorem 3. (Correctness of Channel States). The algorithm initiated by $P_{\text {init }}$ correctly computes channel states using procedure Compute_In-transit_Messages when assuming FIFO channels and using local message counters.

\section{Complexity}

A key feature of our algorithm is that it does not rely on the processes continually and pessimistically reporting their (relevant) activity. The algorithm can be invoked at any arbitrary time, and only the (relevant) activity that occurs in the slice during the algorithm execution needs to be reported. The size of a slice is an important parameter in the complexity of the algorithm. Before embarking on the complexity analysis, we note that Slice_A and Slice_B are both thin slices. We define

- $\widehat{r t t}_{\max }$ to be the expected round-trip transit time between the two farthest nodes in the network.

The expected size of Slice_A and Slice_B is the number of (relevant) events in the execution logs that occur in a duration of time $\widehat{r t t}_{\text {max }}$. With current Internet technology, the round-trip times even across the globe are up to $200 \mathrm{~ms}$. On the other hand, a round-trip communication on an MIMD distributed memory message-passing multiprocessor (e.g., IBM Blue Gene, Cray, Fujitsu, and Hitachi series) using MPI takes microseconds [27].

The following complexity analysis assumes a flat tree overlay topology with the initiator as the root. A similar analysis can be conducted for the ring and more general tree topologies.

Message complexity. From Fig. 2 , it is seen that $6(n-1)$ messages are required across the three phases.

- The total space requirement across all the messages is $O\left(\mid\right.$ Slice $_{-} A|+|$ Slice_$\left._{-} B \mid\right)$.

- If in-transit messages are to be captured, an additional $O\left(n^{2}\right)$ overhead is incurred due to the $n$ Sent and Received vectors.

Message time complexity. Assume that it takes one time unit for a message to reach its destination. The three-phase algorithm (assuming the flat tree topology) takes six time units for the messages in transit. Alternately, this can be expressed as $3 \widehat{r t t}_{\max }$.

Computation time complexity. The computation time complexity is the execution cost. This analysis uses Fig. 5 (and its variant Fig. 9), Fig. 6, and Fig. 10.

\section{Step 1.}

- At the initiator $P_{\text {init }}$, the time complexity is

$$
O\left(\mid \text { Slice_A }_{-}|+| \text {Slice_}_{-} \mid\right) \text {. }
$$

- If in-transit messages are to be reported, an additional $O\left(n^{2}\right)$ overhead is incurred because of arrays Global_Sent and Global_Received.

Step 2.

- At a noninitiator $P_{j}$, the time complexity is

$$
O\left(\left(\mid \text { Slice_}_{-} A|+| \text { Slice_}_{-} B \mid\right) / n\right)
$$

average case.

- If in-transit messages are to be reported, an added $O(n)$ overhead is incurred due to vectors Sent, Received, and Must_Receive.

Step 3: Procedure Compute_Consistent_Snapshot.

- Non-FIFO version, no channel state recording.

- The loop (3a-3i) requires $O\left(1 / n \cdot\left(\mid\right.\right.$ Slice_$_{-} A \mid$. $\mid$ Slice_ $B \mid))$ time. This is because for each process, each of the $1 / n \cdot \mid$ Slice_$_{-} A \mid$ events may have to be examined if they were receive events, and, if so, 
TABLE 3

Complexity of the Snapshot Algorithms, Usable for Stable Property Detection

\begin{tabular}{|c|c|c|}
\hline Metric & $\begin{array}{c}\text { Snapshot Algorithm v.1 } \\
\text { (no channel states recorded) }\end{array}$ & $\begin{array}{l}\text { Snapshot algorithm v.2 } \\
\text { (channel states recorded) }\end{array}$ \\
\hline $\begin{array}{l}\text { Number of messages; } \\
\text { Message space (total); } \\
\text { Message time complexity }\end{array}$ & $\begin{array}{c}6(n-1) \\
O(\mid \text { Slice_A } A) \\
6 \text { hops or } 3 \widehat{r t t}_{\max }\end{array}$ & $\begin{array}{c}6(n-1) \\
O\left(\mid \text { Slice_A|) }+O\left(n^{2}\right)\right. \\
6 \text { hops or } 3 \widehat{r t t}_{\max }\end{array}$ \\
\hline $\begin{array}{l}\text { Computation time } \\
\text { complexity (initiator) }\end{array}$ & $\begin{array}{l}O\left(1 / n \cdot \mid \text { Slice_A }\left.\right|^{2}\right) \text { average } \\
\quad+O(\mid \text { Slice_A } A)+O\left(n^{2}\right)\end{array}$ & $\begin{array}{l}O\left(1 / n \cdot \mid \text { Slice_A }\left.A\right|^{2}\right) \text { average } \\
\quad+O(\mid \text { Slice_A } A)+O\left(n^{2}\right)\end{array}$ \\
\hline $\begin{array}{l}\text { Computation time } \\
\text { complexity (non-initiator) }\end{array}$ & $O(\mid$ Slice_A $A \mid / n)$ average & $O\left(\mid\right.$ Slice $\left.\_A \mid / n\right)$ average $+O(n)$ \\
\hline $\begin{array}{l}\text { Space complexity } \\
\text { (initiator) }\end{array}$ & $O(\mid$ Slice_A $A)$ & $O\left(\left|S l i c e_{-} A\right|\right)+O\left(n^{2}\right)$ \\
\hline $\begin{array}{l}\text { Space complexity } \\
\text { (noninitiator) }\end{array}$ & $O(1 / n \cdot \mid$ Slice_A $\mid)$ average & $O(1 / n \cdot \mid$ Slice_A $A)$ average $+O(n)$ \\
\hline Features & $\begin{array}{c}\text { No inhibition } \\
\text { Application messages unmodified } \\
\text { execution unmodified } \\
\text { no log of history }\end{array}$ & $\begin{array}{c}\text { No inhibition } \\
\text { Application messages unmodified } \\
\text { execution unmodified } \\
\text { no log of history } \\
\text { Sent \& Received vectors per process } \\
\text { Global_Sent, Global_Receive arrays at initiator }\end{array}$ \\
\hline Channels & non-FIFO & FIFO \\
\hline Stable properties detected & Locally stable & All \\
\hline
\end{tabular}

the hash of the message received has to be compared against the hash of the messages sent at each of the possible $\left(1 / n \cdot \mid\right.$ Slice_$\left._{-} B \mid\right)$ events at the sender process. Thus, for each process execution considered from Slice_ $A$, the overhead contributed is $1 / n \cdot \mid$ Slice_A $_{A}|\cdot 1 / n \cdot|$ Slice_$_{B} B \mid$. Across all processes, the overhead becomes $O(1 / n \cdot \mid$ Slice_A $|\cdot|$ Slice_B $B)$ average case.

- Consider the main loop (3m-3z). Observe that each event in Slice $_{-}$A gets examined at most once in line 3o. If a message is sent at this event, due to non-FIFO channels, the hash of this message needs to be compared with the hash of the messages received at possibly each of the events, numbering $1 / n \cdot \mid$ Slice $_{-} A \mid$, at the receiver process. The overhead across all $n$ iterations for 30 to 3 s is $O\left(1 / n \cdot \mid\right.$ Slice $\left.\left._{-} A\right|^{2}\right)$. There is one iteration of lines $3 \mathrm{u}$ to $3 \mathrm{v}$, taking time $O\left(\mid\right.$ Slice $\left._{-} A \mid\right)$. There are at most $n$ iterations of lines $3 \mathrm{x}-3 \mathrm{y}$, costing a total of $O\left(n^{2}\right)$ time. So the time overhead for loop 3m$3 \mathrm{z}$ is $O\left(1 / n \cdot \mid\right.$ Slice_$\left._{A} A\right|^{2}+\mid$ Slice_$\left._{-} A \mid+n^{2}\right)$ average case.

- $\quad$ FIFO version, with recording of channel states

- For the loop in lines $3 a$ to $3 \mathrm{i}$, the overhead is the same as for the non-FIFO version, $O(1 / n$. $\mid$ Slice_A $A \cdot \mid$ Slice_B $\mid)$ average case.

- $\quad$ For the main loop $3 \mathrm{~m}$ to $3 \mathrm{z}$, the overhead is the same as that for the non-FIFO version, namely, $O\left(1 / n \cdot \mid\right.$ Slice_$\left._{-} A\right|^{2}+\mid$ Slice_$\left._{-} A \mid+n^{2}\right)$ average case.

Step 4: Procedure Compute_In-transit_Messages.

- Line $4 \mathrm{a}$ requires $O\left(n^{2}\right)$ time. Lines $4 \mathrm{~b}$ to $4 \mathrm{e}$ require $O\left(\mid\right.$ Slice_$\left._{-} A \mid\right)$ time. The main loop in lines $4 \mathrm{f}$ to $4 \mathrm{k}$ requires $O\left(\mid\right.$ Slice_$_{-} A|+|$ Slice_$\left._{-} B \mid\right)$ time because it requires a single pass through the events of both the slices.
At the initiator $P_{\text {init }}$, the average case computation time complexity for Version 1 (no channel recording) and for Version 2 (channel recording, FIFO channels) is

$$
\begin{aligned}
& O\left(\mid \text { Slice_A } A+\mid \text { Slice_B }_{\mid}\right)+O\left(1 / n \cdot\left(\mid \text { Slice_A }_{\mid}|\cdot| \text { Slice_B }_{\mid} \mid\right)\right) \\
& +O\left(1 / n \cdot \mid \text { Slice_}\left._{\_} A\right|^{2}+\mid \text { Slice_A }_{-} \mid+n^{2}\right) .
\end{aligned}
$$

\section{Space complexity.}

- At initiator.

- The total space complexity is

$$
O(\mid \text { Slice_A } A|+| \text { Slice_B } \mid) \text {. }
$$

- If in-transit messages are recorded, $O\left(n^{2}\right)$ additional overhead is incurred for arrays Global_ Sent and Global_Received.

- At noninitiator:

- $\quad$ The average space complexity is

$$
\left(1 / n \cdot \max \left(\mid \text { Slice_A }_{-}|,| \text {Slice_B }_{-} \mid\right)\right)
$$

for the $\log$ of the slice.

- If channel states are recorded, $O(n)$ additional space is required for the Sent and Received vectors.

The above analysis considered Slice_A and Slice_ $B$ separately. Note that Slice_$_{-} A$ and Slice $_{-} B$ are thin slices and are expected to have similar size. Assuming $\mid$ Slice_$_{-} A \mid=$ $\mid$ Slice_$B \mid$, Table 3 summarizes the complexity results for the proposed algorithm.

\section{Detecting Stable Predicates}

Theorems 2 and 3 showed the safety of the snapshot algorithm. The proposed algorithm Version 2 to record a consistent global state can be used in a straightforward manner to detect any stable predicate. Each process records 
the (possibly changing) values of the variables over which the predicate is defined in Slice_A. When the initiator computes the consistent cut $S$, it can also evaluate the predicate over these variables after cut $S$. If the predicate evaluates to true, then it is true and remains true henceforth because it is stable. Safety of the predicate detection follows from the correctness of Theorems 2 and 3 and the definition of a stable predicate. A similar argument holds for Version 1 of the algorithm which detects any locally stable predicate. We now analyze the liveness of both versions of the algorithm.

- Version 1 can detect locally stable predicates. A locally stable predicate can be evaluated over the variables at the processes. Liveness follows from the fact that if a locally stable predicate becomes true after some cut $Q$, then any invocation of the snapshot algorithm which computes a cut $S$ such that $Q \subseteq S$ will detect the locally stable predicate.

- Version 2 can detect any stable predicate. If a stable predicate is true in $S$, it will be detected because the entire system state (processes and channels) is observable at $S$. Specifically, liveness follows from the fact that if a stable predicate becomes true in some global state $Q$, then any invocation of the snapshot algorithm which computes a state $S$ such that $Q \subseteq S$ will detect the stable predicate.

\section{Algorithm Variants}

Our algorithm V2 used the Sent and Received vectors to compute the channel states in the "Reduce A" approach. This section gives two variants of V2. The first variant V2.A uses the Sent and Received vectors to also construct the consistent cut $S$. The second variant V2.B uses the "Extend Z" approach to construct the consistent cut. V2.A has somewhat better complexity than V2. V2.B has a higher complexity than V2 but is given to demonstrate the complementary approach to the "Reduce A" approach.

\subsection{Reducing the "Second Cut" Using Counters (v.2.A)}

Version 2 constructed $S$ using the idea of detecting inconsistencies by tracing through Slice_A (see Fig. 6). The Sent and Received vectors are required to capture in-transit messages. However, these vectors can also be used for constructing the consistent cut $S$-the algorithm is very similar to that in Fig. 6, Section 3, and, hence, the repeat loop has at most $n$ iterations. The algorithm is given in Fig. 12. Global_Sent $[i, \tilde{i}]$ and Global Received $[i, \tilde{i}]$ get updated to their values for event $\star L a s t \_S[i]$, in lines $3 \mathrm{~A}$ and $3 \mathrm{u}$, respectively. Hence, Step 4 gets simplified from that in Fig. 10.

The Global_Received is initialized at the end of Phase I, whereas the Global_Sent is initialized at the end of Phase II. In lines $3 \mathrm{a}$ to $3 \mathrm{~g}, S_{\max }$ is constructed and Global_Received is modified (line $3 \mathrm{e}$ ) to reflect the receive count at $S_{\max }$. Lines $3 \mathrm{~h}$ to $3 \mathrm{k}$ modify Global_Sent to reflect the send count at $S_{\text {max }}$. Lines 31 to 3 initialize Last_T, Last_V, and Last_S. The main repeat loop $(3 \mathrm{o}-3 \mathrm{H})$ achieves the counterpart of lines $3 \mathrm{~m}$ to $3 \mathrm{z}$ of Fig. 6 . As the code iterates to construct $S$ from $S_{\text {max }}$, so also in each iteration, Global_Received is decremented in line $3 \mathrm{u}$ to reflect the receive count of $S$ for that iteration of the repeat loop. Analogously, the Global_Sent count is decremented in line 3A to reflect the send count of $S$ in that iteration of the repeat loop.

Complexity. The complexity for all the metrics except the computation time complexity is the same as for the algorithm v.2 in Fig. 6 (see Table 3). Lines 3a to 3g construct $S_{\text {max }}$ - the initiator can detect a message received at an event in Slice_A $_{A}$ as being sent from an event in Slice_$_{-} B$ in $O\left(\mid\right.$ Slice $\left._{-} A \mid\right)$ time. Lines 3h-3k which update Global_Sent for this $S_{\text {max }}$ also take $O\left(\mid\right.$ Slice $\left._{-} A \mid\right)$ time. Across all iterations of the repeat loop, the number of times event $y$ is enumerated in line $3 q$ is at most $\mid$ Slice_A $_{A} \mid$, and the number of times event $z$ is enumerated in line $3 \mathrm{~s}$ is at most $\mid$ Slice_A $A$. Similarly, across all iterations of the repeat loop, the number of times event $y$ is enumerated in line $3 y$ is at most $\mid$ Slice_A $A \mid$. The lines 3B-3D are executed once, leading to $\mid$ Slice_$_{-} A \mid$ overhead. Lines 3F-3G contribute $O\left(n^{2}\right)$ overhead across the maximum of $n$ executions of the repeat loop. Hence, the time complexity of Step 3 is $O\left(\mid\right.$ Slice $\left._{-} A \mid+n^{2}\right)$. The time complexity of Step (4) is $O\left(\mid\right.$ Slice $_{-} A|+|$ Slice_$\left._{-} B \mid\right)$. Assuming $\mid$ Slice $_{-} A|=|$ Slice $_{-} B \mid$, the time complexity of the algorithm is $O\left(\mid\right.$ Slice_A $\left.A \mid+n^{2}\right)$.

\subsection{Extending the "First Cut" (v.2.B)}

Using the Sent and Received vectors that are required to capture the in-transit messages, it is possible to construct the consistent cut $S$ using the "extend $Z$ " approach described in Section 3.1.

- Steps 1 and 2 will differ from Fig. 9 as follows: The Sent vectors will be reported in the Phase I reply (i.e., for cut $Z$ ) instead of in the Phase II reply.

- The modified Step 3 is described in Fig. 13. Step 4 is the same as in Fig. 12.

In Step 3, cuts $S$ and $V$ are initialized to $Z$ in lines 3a to $3 \mathrm{~b}$. Each iteration of the repeat loop adds the minimum prefix from $S l i c e_{-} A \backslash S$ to $S$, to remove any inconsistency by including necessary send events (lines $3 \mathrm{~d}$ to $3 \mathrm{i}$ ). However, when such a prefix is added to $S$, some receive events may also be included such that the corresponding send events are not included in the prefixes added in this iteration. Thus, further iterations of the repeat loop are needed until $S$ does not change in an entire iteration (line $3 n$ ). Last_V is a working variable used to track the current greatest lower bound on the source of inconsistency in an iteration. Global_Sent $[i, \tilde{i}]$ and Global_Received $[i, \tilde{i}]$ get updated to their values for event $\star L a s t \_S[i]$, in lines $3 \mathrm{~h}$ and $3 \mathrm{~m}$, respectively. Hence, Step 4 gets simplified from that in Fig. 10, and is the same as shown in Fig. 12. The correctness proof is analogous to that for the algorithm presented in Sections 3 and 4.

Complexity. For the "Extend $Z$ " approach, the complexity for all the metrics except the computation time complexity is the same as for the "Reduce $A$ " approach (see Algorithm v.2 in Table 3).

It can be seen that there are at most $n$ iterations of the main repeat loop in Fig. 13. In each iteration,

- $\quad$ Slice $A$ is examined in lines $3 \mathrm{~d}$ to $3 \mathrm{i}$, leading to $O(\mid$ Slice_A $\mid)$ time overhead.

- Lines 3n and 3r contribute $O(n)$ time overhead. 
(3) Process $P_{\text {init }}$ executes Compute_Consistent_Snapshot. Last_S $_{\text {max }}$, Last_S, Last_T, Last_V: array of pointers to event; found: boolean;

// latest event to construct $S_{\max }$ at a process

(3a) for $i=1$ to $n$ do

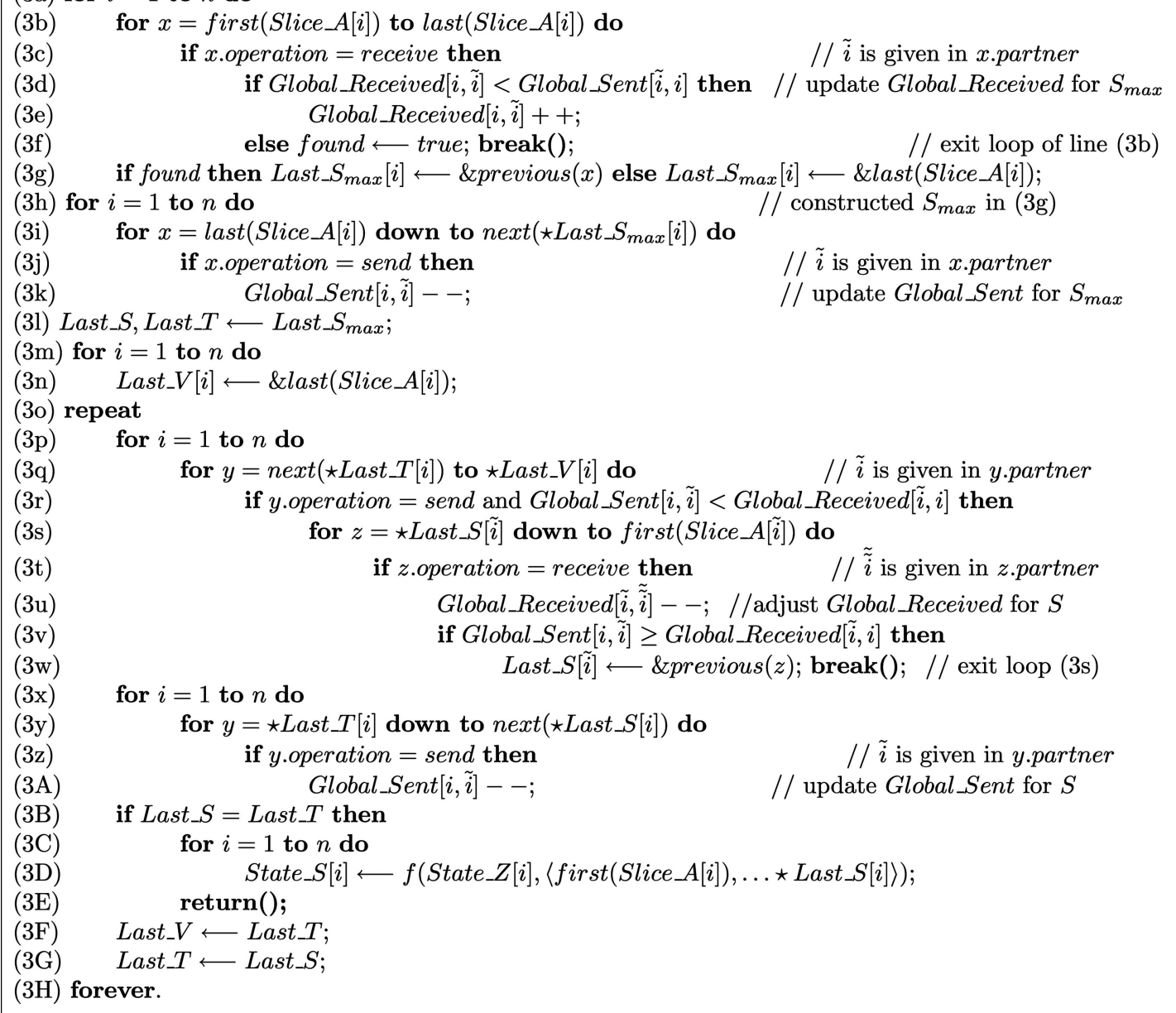

(4) Process $P_{\text {init }}$ executes Compute_In-transit_Messages(Last_S).

(4a) $\forall i \forall j$, transit $(\star$ Last_S $[i], \star$ Last_S $[j]) \longleftarrow \emptyset$;

(4b) for $j=1$ to $n$ do

(4c) for $x=\operatorname{next}\left(\star L a s t \_S[j]\right)$ to $\operatorname{last}($ Slice_B $[j])$ do

(4d) if x.operation = receive then $\quad / / \tilde{j}$ is given in $x . p a r t n e r$

(4e) Global_Received $[j, \tilde{j}]++$;

(4f) $\quad$ if Global_Sent $[\tilde{j}, j] \geq$ Global_Received $[j, \tilde{j}]$ then

(4ransit $\left(\star\right.$ Last $_{-} S[\tilde{j}], \star$ Last_S$\left.[j]\right) \longleftarrow$ transit $\left(\star L a s t \_S[\tilde{j}], \star L a s t \_S[j]\right) \cup\{x . M\}$

Fig. 12. Version V2A: Constructing consistent cut $S$ for the Version 2 (FIFO channels) algorithm using Global_Sent and Global_Received.

Lines 30 to $3 q$, which are executed only once, contribute $O\left(\left|S_{\text {Slice }} A\right|\right)$ time overhead. Lines $3 \mathrm{j}$ to $3 \mathrm{~m}$ contribute $O\left(\left|S_{\text {Slice }} A\right|\right)$ time overhead across all iterations. So the execution time complexity at the initiator is

$$
O\left(n \cdot \mid \text { Slice_}_{-} A \mid+n^{2}\right) .
$$

\subsection{Complexity Trade-offs}

The complexity of the three snapshot algorithms- "Reduce $A$ " without arrays, (Fig. 6), "Reduce $A$ " with arrays (Fig. 12), and "Extend $Z$ " with arrays, (Fig. 13)-is the same for all metrics except the time computation complexity at the initiator. This computation time complexity is compared in Table 4. The $O\left(n^{2}\right)$ is a lower bound because the states of $O\left(n^{2}\right)$ channels have to be recorded.

Let $w$, termed the slice width, be the average number of events at a process in a slice, i.e., $w=\mid$ Slice_A $A_{\mid} / n$. The width $w$ depends on the geographical span of the network as well as the expected rate of generation of relevant events. For thin slices and large systems, it is likely that $w<n$. The computation time complexity of the algorithms in terms of $w$ is also given in Table 4 . In order to remain bounded by $O\left(n^{2}\right)$, the relationship between $O(w)$ and $O(n)$ is also expressed. The algorithm in Fig. 12 provides the best flexibility. The algorithm using the "Extend Z" approach has higher complexity but is given for completeness as it complements the "Reduce A" approach. 


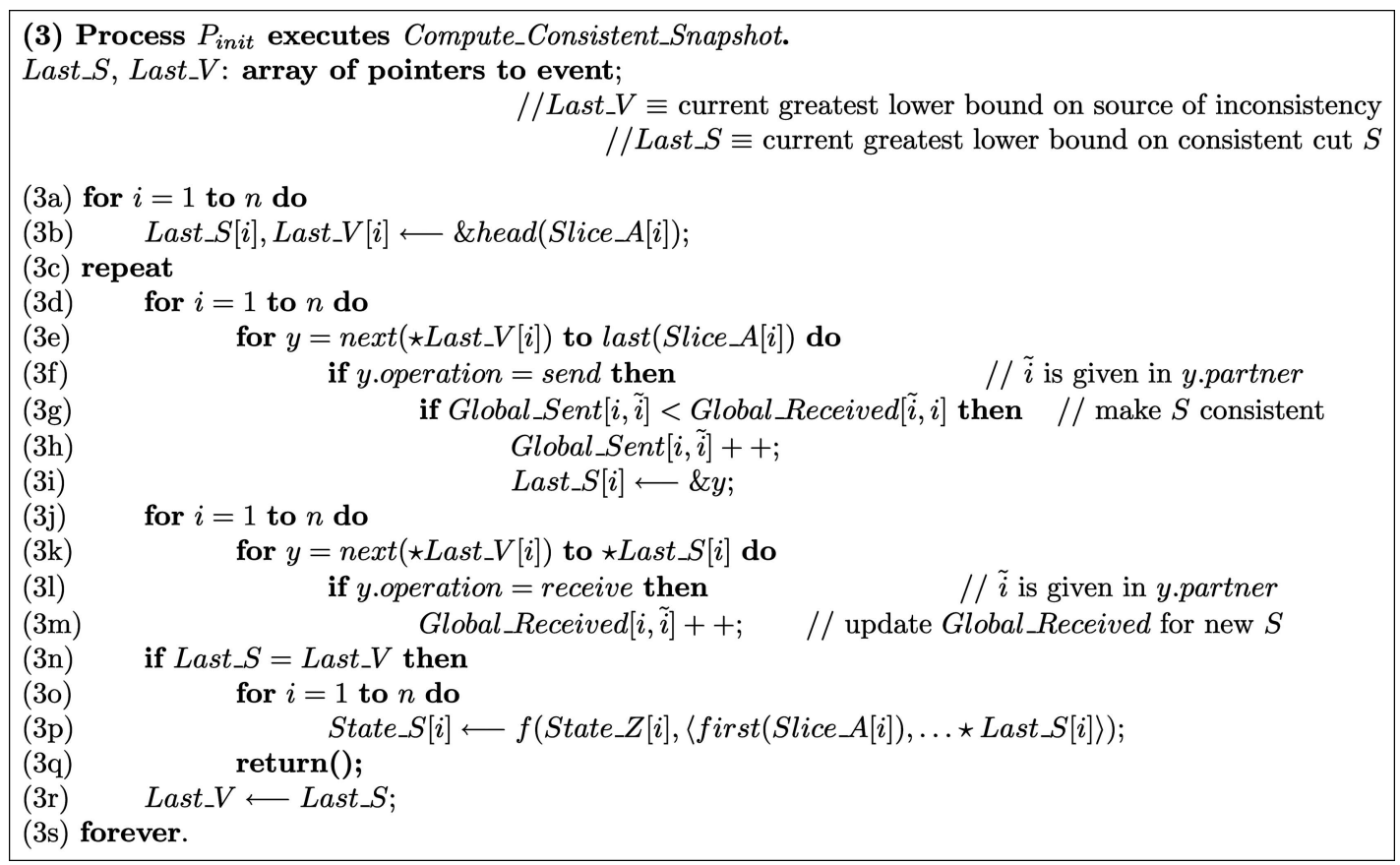

Fig. 13. Iteratively constructing a consistent cut using the "Extend $Z$ " approach.

TABLE 4

Computation Time Complexity of the Snapshot Algorithms That Record States of FIFO Channels

\begin{tabular}{|l||l|l|}
\hline $\begin{array}{l}\text { Snapshot Algorithm v.2 } \\
\text { ("Reduce } A \text { ", Figure 6) }\end{array}$ & $\begin{array}{l}\text { Snapshot algorithm v.2.A } \\
\text { ("Reduce } A \text { ", Figure 12) }\end{array}$ & $\begin{array}{l}\text { Snapshot algorithm v.2.B } \\
\text { ("Extend Z", Figure 13) }\end{array}$ \\
\hline \hline$O\left(1 / n \cdot \mid\right.$ Slice_A $\left.\left.A\right|^{2}\right)+O(\mid$ Slice_A $A \mid)+O\left(n^{2}\right)$ & $O\left(\left|S l i c e \_A\right|\right)+O\left(n^{2}\right)$ & $O\left(n \cdot \mid\right.$ Slice_A|) $+O\left(n^{2}\right)$ \\
$=O\left(1 / n \cdot(n w)^{2}\right)+O(n w)+O\left(n^{2}\right)$ & $=O(n w)+O\left(n^{2}\right)$ & $=O(n \cdot n w \mid)+O\left(n^{2}\right)$ \\
$=O\left(n w^{2}+n^{2}\right)$ & $=O\left(n w+n^{2}\right)$ & $=O\left(n^{2} w+n^{2}\right)$ \\
$=O\left(n^{2}\right)$ if $O(w)<O(\sqrt{n})$ & $=O\left(n^{2}\right)$ if $O(w)<O(n)$ & $=O\left(n^{2} w\right)$ \\
\hline
\end{tabular}

\subsection{Two-Phase Algorithm: Local States under FIFO Channels, V2.C}

Observe that the modified code in Fig. 12 to construct $S_{\max }$ using Global_Sent and Global_Received does not use Slice_B. This immediately suggests a two-phase algorithm to record local states consistently if each process maintains the Sent and Received counters. The two-phase exchange would be as shown in Fig. 9-only lines $1 \mathrm{~g}$ to $1 \mathrm{i}$ and $2 \mathrm{f}$ to $2 \mathrm{~h}$ would be omitted. The cost would be the same as for the snapshot algorithm v.2 (see Table 3) except that

- Number of messages $=4(n-1)$, message time complexity is four hops or $2 \widehat{r t t}_{\max }$ and locally stable properties are detected.

\section{Discussion}

This paper first presented two algorithms to detect stable properties. The first algorithm can be used to detect any locally stable properties in a non-FIFO system, whereas the second algorithm can detect any stable property in a FIFO system, under the assumptions P1-P4, Section 1. A simple and elegant three-phase strategy of uncoordinated observation of local states was used to create a consistent distributed snapshot. The first snapshot algorithm computed consistent process states without requiring FIFO channels. The second algorithm computed process states and channel states consistently but required FIFO channels. A key feature of our algorithms is that they do not rely on the processes continually and pessimistically reporting their (relevant) activity. The algorithms can be invoked at any arbitrary time, and only the (relevant) activity that occurs in the thin slice during the algorithm execution needs to be reported. The paper then showed three variants of the algorithms, that adopt a complementary approach to the originally presented algorithms.

The goal of procedure Compute_Consistent_Snapshot resembles the goal of finding a consistent rollback state when using asynchronous checkpointing [24]. The idea in both is to construct a consistent state out of an inconsistent state, given the logs of the execution. However, the approaches, algorithms, and purposes are different. Further, our approach also does not require logging the entire history of the execution, does not modify application messages by tagging sequence numbers, and does not use a reactive event model for the execution.

The snapshot algorithms presented here create an ondemand thin slice of the execution that is guaranteed to contain a consistent cut. The "thin slice" approach involves very low overhead for the algorithm when channel states are not needed. The proposed snapshot algorithm that also computes channel states requires $O\left(n^{2}\right)$ time and space at the initiator, which can be viewed as a drawback. However, note that in practice, this is preferable to existing algorithms that require $O\left(n^{2}\right)$ messages instead of $O(n)$ messages. The 
proposed algorithms also have a snapshot readily available at the initiator, whereas most of the existing algorithms record a distributed snapshot and need to incur an additional cost to collect it.

The proposed algorithms record some consistent global state in Slice_A. In order to construct a global state that contains a particular local state at the initiator, the following can be done. In the Phase II recordings, the initiator needs to ensure that it records its local state before any other process. (We leave the correctness reasoning to the reader). The initiator still needs to have initiated Phase I earlier, which means that if a look-ahead is not possible, the initiator needs to freeze its execution between its Phase I and Phase II recordings.

\section{APPENDIX A}

\section{Correctness Proof}

\section{A.1 Consistency}

Lemma 1. In each iteration of the repeat loop of lines $3 m$ to $3 z$ of Compute_Consistent_Snapshot in Fig. 6, only the minimum suffix from the cut identified by Last_S that is needed to remove inconsistency-causing messages is deleted.

Proof Sketch. This is evident from lines $3 \mathrm{n}$ to $3 \mathrm{~s}$, where Last_S $[i]$ is adjusted to point to the earlier of 1 ) the current event being pointed to and 2) the event immediately preceding the receipt of a message that was sent after the event pointed to by Last_T $[i]$. Note that Last_T $[i]$ and Last_S $[i]$ are the same at the start of each iteration; hence any message identified in event 2 causes the state identified by $S$ to be inconsistent. This adjustment in line 3 s eliminates the minimum suffix from the local state identified by Last_S$_{-}[i]$ in order to eliminate the receive event and restore consistency (with respect to this inconsistency-causing message).

Denote a message $m^{i}$ by its send and receive events $\left\langle s^{i}, r^{i}\right\rangle$. A message chain is a causal sequence of messages $\left\langle m^{1}, m^{2} \ldots m^{k}\right\rangle$ such that $r^{i} \prec s^{i+1}$, for $i \in[1 \ldots(k-1)]$, and events $r^{i}$ and $s^{i+1}$ must occur at the same process. For simplicity, we denote the cut $S$ by the pointer array Last_S. An inconsistency-causing message chain is such that $s^{1} \notin$ Last_S and $r^{1} \in L_{\text {Lst_S }}$, and, for all $j \in[2 \ldots k]$, we also have $s^{j}, r^{j} \in$ Last_S.

Lemma 2. For any inconsistency-causing chain $M$ given by $\left\langle m^{1}, m^{2}, \ldots m^{k}\right\rangle$, after $j$ iterations of the repeat loop in lines $3 m$ to $3 z$ in Fig. 6, the chain becomes $M^{\prime}$ given by $\left\langle m^{j+1} \ldots m^{k}\right\rangle$, where $s^{j+1} \notin$ Last_S.

Proof Sketch. We show this by induction on the number of iterations of the repeat loop.

Case $\mathbf{j}=\mathbf{1}$. From Lemma 1, only the minimal inconsistency-causing suffix due to the receive event $r^{1}$ is removed. As $r^{1} \prec s^{2}$, we therefore have the chain $\left\langle m^{2}, m^{3} \ldots m^{k}\right\rangle$ where $s^{2} \notin$ Last_S and all other send and receive events in the inconsistency-causing chain are in Last_S.

Case $\mathbf{j}=\mathbf{x}$. Assume that the hypothesis is true for any value of $j$. So, after $x$ iterations, we have the inconsistency-causing chain $\left\langle m^{x+1} \ldots m^{k}\right\rangle$, where $s^{x+1} \notin$ $L a s t_{-} S$ and all other send and receive events in the inconsistency-causing chain are in Last_S.
Case $\mathbf{j}=\mathbf{x}+\mathbf{1}$. From the induction hypothesis, we have the inconsistency-causing chain $\left\langle m^{x+1} \ldots m^{k}\right\rangle$, where $s^{x+1} \notin$ Last_S and all other send and receive events in the inconsistency-causing chain are in Last_S. From Lemma 1, only the minimal inconsistency-causing suffix due to the receive event $r^{x+1}$ is removed. As $r^{x+1} \prec s^{x+2}$, we therefore have the inconsistency-causing chain

$$
\left\langle m^{x+2}, m^{x+3} \ldots m^{k}\right\rangle,
$$

where $s^{x+2} \notin$ Last_S and all other send and receive events in the inconsistency-causing chain are in Last_S. $\square$

Lemma 3. The longest inconsistency-causing chain has length $n-1$.

Proof Sketch. We prove by contradiction. Let the last message in the chain be $m^{k}, k>n-1$. As the longest inconsistency-causing chain has length $n$ or greater than $n$, then there is at least one process that has a receive event $r^{k}$ that happens later than $s^{h}$ at the same process, i.e., $m^{h}$ was sent earlier than receiving $m^{k}$. As the send event $s^{h}$ happened before $r^{k}$ and $s^{h}$ itself did not belong to the cut marked by Last_S in iteration $h$, we have that $r^{k}$ also cannot belong to the cut Last_S. Hence, $m^{k}$ does not satisfy the condition of being a part of an inconsistency-causing chain.

Theorem 1. Procedure Compute_Consistent_Snapshot in Fig. 6, terminates in $n$ iterations of the repeat loop of lines $3 m$ to $3 z$.

Proof Sketch. Follows from Lemmas 1, 2, and 3. The longest inconsistency-causing chain has $n-1$ messages. After $n-1$ iterations, there is no inconsistency-causing chain. An additional iteration is required to detect that Last_T = Last_S (line 3t) and then to exit the repeat loop.

Theorem 2 (Correctness of Local Recordings). The algorithm initiated by $P_{\text {init }}$ terminates by finding a consistent cut $S$, where $S\left(t_{A_{-} \text {start }}\right) \subseteq S \subseteq S_{\max }$.

Proof Sketch. Steps 1 and 2 of the algorithm contain a threephase exchange, which can be seen to terminate. Specifically, the only waiting is for sent messages to be received, and assuming a reliable system, this waiting is finite in an asynchronous system. From Theorem 1, Step 3, Compute_Consistent_Snapshot, also terminates. Step 4, Compute_In-transit_Messages, does local processing and can be seen to terminate. Hence, the algorithm terminates.

The Compute_Consistent_Snapshot procedure initializes $S$ to $S_{\max }$. The only operations on $S$ in each iteration are the deletion of a suffix. Hence, on termination, $S \subseteq S_{\max }$.

There are a bounded number of events in $S_{\max } \backslash$ $S\left(t_{A_{-} s t a r t}\right)$ and in each iteration that does not result in termination, at least one event is removed. From Lemma 1, only the minimum suffix that causes inconsistency is deleted at each step. Hence, in a finite number of steps, either $S\left(t_{A_{\_} s t a r t}\right)$ or some cut $S$, where $S\left(t_{A_{-} \text {start }}\right) \subset S$, is constructed such that no process would have a suffix deleted. This would be a consistent cut. The theorem now follows. 


\section{A.2 Recording Channel States}

Theorem 3 (Correctness of Channel States). The algorithm initiated by $P_{\text {init }}$ correctly computes channel states using procedure Compute_In-transit_Messages when assuming FIFO channels and using local message counters.

Proof Sketch. For channel $C_{i, j}$, the in-transit messages are those sent up to event $\star L a s t \_S[i]$ and received after event $\star$ Last_S $[j]$. Observe from Step $2 \mathrm{~g}^{\prime}$ of Fig. 9 that all messages sent up to $\star L a s t_{-} S[i]$ are guaranteed to be received by $\operatorname{last}\left(\right.$ Slice_$\left._{-} B[i]\right)$, if channels are FIFO. Observe from loop $4 \mathrm{f}-4 \mathrm{k}$ that the channel state is computed as per (2). All messages sent up to $\star L a s t \_S[i]$ and received after $\star L a s t_{-} S[j]$ are therefore identified as belonging to the channel state using the local message counters.

\section{ACKNOWLEDGMENTS}

The authors thank the referees for their valuable comments that have significantly simplified the organization and presentation of the results. A preliminary conference version of this result appears in [13].

\section{REFERENCES}

[1] A. Acharya and B.R. Badrinath, "Recording Distributed Snapshots Based on Causal Order of Message Delivery," Information Processing Letters, vol. 44, no. 6, pp. 317-321, 1992.

[2] S. Alagar and S. Venkatesan, "An Optimal Algorithm for Distributed Snapshots with Causal Message Ordering," Information Processing Letters, vol. 50, no. 6, pp. 311-316, 1994.

[3] R. Atreya, N. Mittal, and V.K. Garg, "Detecting Locally Stable Predicates without Modifying Application Messages," Proc. Int'l Conf. Principles of Distributed Systems, pp. 20-33, 2003.

[4] K.M. Chandy and L. Lamport, "Distributed Snapshots: Determining Global States of Distributed Systems," ACM Trans. Computer Systems, vol. 3, no. 1, pp. 63-75, 1985.

[5] C. Critchlow and K. Taylor, "The Inhibition Spectrum and the Achievement of Causal Consistency," Distributed Computing, vol. 10, no. 1, pp. 11-27, 1996.

[6] C.J. Fidge, "Logical Time in Distributed Computing Systems," Computer, vol. 24, no. 8, pp. 28-33, 1991.

[7] J.-M. Helary, "Observing Global States of Asynchronous Distributed Applications," Proc. Int'l Workshop Distributed Algorithms, pp. 124-125, 1989.

[8] J. Helary, C. Jard, N. Plouzeau, and M. Raynal, "Detection of Stable Properties in Distributed Applications," Proc. ACM Symp. Principles of Distributed Computing, pp. 125-136, 1987.

[9] J. Helary and M. Raynal, "Towards the Construction of Distributed Detection Programs, with an Application to Distributed Termination," Distributed Computing, vol. 7, pp. 137-147, 1994.

[10] G.S. Ho and C.V. Ramamoorthy, "Protocols for Deadlock Detection in Distributed Database Systems," IEEE Trans. Software Eng., vol. 8, no. 6, pp. 554-557, 1982.

[11] A. Kshemkalyani, M. Raynal, and M. Singhal, "An Introduction to Snapshot Algorithms in Distributed Computing," Distributed Systems Eng., vol. 2, no. 4, pp. 224-233, 1995.

[12] A. Kshemkalyani and M. Singhal, "Correct Two-Phase and OnePhase Deadlock Detection Algorithms for Distributed Systems," Proc. IEEE Symp. Parallel and Distributed Processing, pp. 126-129, 1990.

[13] A. Kshemkalyani and B. Wu, "Nonintrusive Snapshots Using Thin Slices," Proc. Int'l Conf. Embedded and Ubiquitous Computing, pp. 572-583, 2005.

[14] T.-H. Lai and T. Yang, "On Distributed Snapshots," Information Processing Letters, vol. 25, no. 3, pp. 153-158, 1987.

[15] L. Lamport, "Time, Clocks, and the Ordering of Events in a Distributed System," Comm. ACM, vol. 21, no. 7, pp. 558-565, 1978.
[16] H.F. Li, T. Radhakrishnan, and K. Venkatesh, "Global State Detection in Non-FIFO Networks," Proc. IEEE Int'l Conf. Distributed Computing Systems, pp. 364-370, 1987.

[17] K. Marzullo and L.S. Sabel, "Efficient Detection of a Class of Stable Properties," Distributed Computing, vol. 8, no. 2, pp. 81-91, 1994.

[18] F. Mattern, "Virtual Time and Global States of Distributed Systems," Proc. Workshop Parallel and Distributed Algorithms, pp. 215-226, 1989.

[19] F. Mattern, "Efficient Algorithms for Distributed Snapshots and Global Virtual Time Approximation," J. Parallel Distributed Computing, vol. 18, no. 4, pp. 423-434, 1993.

[20] M. Raynal, Distributed Algorithms and Protocols. Wiley and Sons, 1988.

[21] A. Schiper and A. Sandoz, "Strong Stable Properties in Distributed Systems," Distributed Computing, vol. 8, no. 2, pp. 93-103, 1994.

[22] M. Spezialetti and P. Kearns, "Efficient Distributed Snapshots," Proc. IEEE Int'l Conf. Distributed Computing Systems, pp. 382-388, 1986.

[23] N. Sridhar and P. Sivilotti, "Lazy Snapshots," Proc. Parallel and Distributed Computing Systems, pp. 96-101, 2002.

[24] S. Venkatesan and T.-Y. Juang, "Efficient Algorithms for Optimistic Crash Recovery," Distributed Computing, vol. 8, no. 2, pp. 105-114, 1994.

[25] S. Venkatesan, "Message-Optimal Incremental Snapshots," Proc. IEEE Int'l Conf. Distributed Computing Systems, pp. 53-60, 1989.

[26] M. Weiser, "Programmers Use Slices When Debugging," Comm. ACM, vol. 25, no. 7, pp. 446-452, 1982.

[27] “Top 500 Supercomputer Sites," http://www.top500.org, 2006

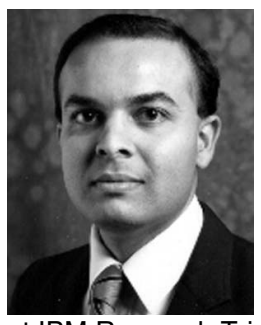

Ajay Kshemkalyani received the BTech degree in computer science and engineering from the Indian Institute of Technology, Bombay, in 1987 and the $\mathrm{PhD}$ degree in computer and information science from the Ohio State University in 1991. His research interests are in computer networks, distributed computing, algorithms, and concurrent systems. He has been an associate professor at the University of Illinois at Chicago since 2000, before which he spent several years at IBM Research Triangle Park working on various aspects of computer networks. He is a member of the ACM and a senior member of the IEEE. In 1999, he received the US National Science Foundation's CAREER Award. He is currently on the editorial board of the Elsevier journal Computer Networks.

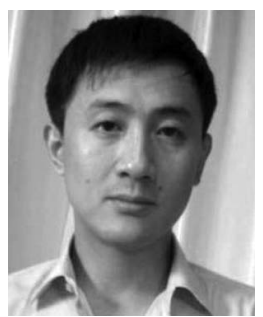

Bin $\mathrm{Wu}$ received the $\mathrm{BS}$ and $\mathrm{MS}$ degrees from the Department of Energy Engineering at Zhejiang University, China, in 1992 and 1995, respectively. He received the MS degree in computer science from the University of Illinois at Chicago in 2002. He is now working toward the $\mathrm{PhD}$ degree in computer science at the University of Illinois at Chicago. His research interests include distributed computing, peer-topeer networks, protocols and algorithms, and Web-based healthcare systems.

$\triangleright$ For more information on this or any other computing topic, please visit our Digital Library at www.computer.org/publications/dlib. 